Comment. Math. Helv. 72 (1997) 167-202

(C) 1997 Birkhäuser Verlag, Basel

$0010-2571 / 97 / 020167-36 \$ 1.50+0.20 / 0$

Commentarii Mathematici Helvetici

\title{
Tilings and finite energy retractions of locally symmetric spaces
}

Leslie Saper

\begin{abstract}
Let $\Gamma \backslash \bar{X}$ be the Borel-Serre compactification of an arithmetic quotient $\Gamma \backslash X$ of a symmetric space of noncompact type. We construct natural tilings $\Gamma \backslash \bar{X}=\coprod_{P} \Gamma \backslash \bar{X}_{P}$ (depending on a parameter b) which generalize the Arthur-Langlands partition of $\Gamma \backslash X$. This is applied to yield a natural piecewise analytic deformation retraction of $\Gamma \backslash \bar{X}$ onto a compact submanifold with corners $\Gamma \backslash X_{0} \subset \Gamma \backslash X$. In fact, we prove that $\Gamma \backslash X_{0}$ is a realization (under a natural piecewise analytic diffeomorphism) of $\Gamma \backslash \bar{X}$ inside the interior $\Gamma \backslash X$. For application to the theory of harmonic maps and geometric rigidity, we prove this retraction and diffeomorphism have finite energy except for a few low rank examples. We also use tilings to give an explicit description of a cofinal family of neighborhoods of a face of $\Gamma \backslash \bar{X}$, and study the dependance of tilings on the parameter $\mathbf{b}$ and the degeneration of tilings.
\end{abstract}

Mathematics Subject Classification (1991). Primary 22E40, 53C35 (Secondary 58E20).

Keywords. Borel-Serre compactification, corners, locally symmetric space, tiling, finite energy retraction, harmonic map.

\section{Introduction}

Let $X=G / K$ be a symmetric space of noncompact type and let $\Gamma \subset G$ be a discrete arithmetic group of isometries. Suppose that $\Gamma \backslash X$ is noncompact. In order to introduce the main subject of this paper, tilings of locally symmetric spaces, we first consider the following problem: find an explicit deformation retract $r^{\prime}$ : $\Gamma \backslash X \rightarrow \Gamma \backslash X_{0} \subset \Gamma \backslash X$ which has compact image. Equivalently, find a $\Gamma$-equivariant retract $r: X \rightarrow X_{0}$ where $X_{0} \subseteq X$ is $\Gamma$-invariant and compact modulo $\Gamma$.

One approach is given by Raghunathan [40] (see also Harder [27]), who constructs a smooth function $h: \Gamma \backslash X \rightarrow \mathbb{R}$ with compact sublevel sets and with no

This research was supported in part by NSF Grants DMS-8957216 and DMS-9100383, a grant from The Duke Endowment, and an Alfred P. Sloan Research Fellowship. During the final revision, the author enjoyed the hospitality of the Katholischen Universität Eichstätt as the Hermann-Minkowski Gastprofessur sponsored by the Maximilian-Bickhoff-Stiftung. 
critical points outside a compact subset. A retraction is then obtained by flowing backwards along the gradient field of $h$. Moreover, one can use $h$ to compactify $\Gamma \backslash X$ by attaching a smooth boundary corresponding to $h=\infty$. But for many applications this is insufficient since $h$ and the retract are neither very explicit nor canonical.

To explain our approach, we recall the construction of the more natural compactification of Borel and Serre [12] in which $\Gamma \backslash X$ is realized as the interior of a real analytic manifold with corners $\Gamma \backslash \bar{X}$. To every parabolic $\mathbb{Q}$-subgroup $P \subseteq G$ there is associated a free geodesic action of a torus $A_{P} \cong\left(\mathbb{R}^{>0}\right)^{r}$ on $X$ and a subgroup ${ }^{0} P \subset P$ whose orbits (called canonical cross-sections) are orthogonal to the geodesic action. Thus there is a canonical decomposition $X \cong A_{P} \times e(P)$ (depending only a choice of basepoint) where $e(P)$ is a homogeneous space for ${ }^{0} P$. Now the construction of $\bar{X}$ proceeds in three steps:

(1) Enlarge $A_{P}$ to $\bar{A}_{P} \cong\left(\mathbb{R}^{>0} \cup\{\infty\}\right)^{r}$ - a model "corner".

(2) Use $\bar{A}_{P}$ and the above decomposition to induce a partial bordification associated to $P$, namely, $X \cong A_{P} \times e(P) \subseteq \bar{A}_{P} \times e(P) \equiv X(P)$.

(3) For $P \subseteq R$, there is a natural inclusion $X(R) \subseteq X(P)$ as an open submanifold with corners; let $\bar{X}$ be the union of the bordifications $X(P)$ associated to all $P$.

In other words, $\bar{X}$ has been formed from $X$ by (for each $P$ ) going to $\{\infty\}^{r}$ under the geodesic action of $A_{P}$ and there attaching a copy of $e(P)$ as a codimension $r$ boundary face.

Thus the corners of $\bar{X}$ are a reflection of geometric structure that exists in the interior, and we want a retract that extends to $\bar{X}$ and preserves this structure. In particular, $X_{0}$ should also be a manifold with corners whose boundary faces $\partial^{P} X_{0}$ lie in canonical cross-sections $\left\{b_{P}\right\} \times \underline{e(P)}$. Here lies much of the difficulty of our problem: a tubular neighborhood of $\overline{e(P)}$ in $\bar{X}$ would easily allow one to move $\overline{e(P)}$ into the interior. But the natural tubular neighborhood $(a, \infty]^{r} \times e(P)$ of $e(P)$ in $X(P)$ does not in general extend to a tubular neighborhood of $\overline{e(P)}$ in $\bar{X}$. This is because in general the canonical functions on $X \cong A_{P} \times e(P)$ induced by the coordinates on $A_{P}$ (given by roots) do not agree with those for a smaller parabolic.

Nonetheless such retractions exist. Their existence is equivalent to the existence of certain decompositions $\bar{X}=\coprod_{P} \bar{X}_{P}$ which we will call tilings and which will be our primary object of study. These tilings are characterized by certain simple properties and behave naturally under automorphisms of $G$. In this paper we will first define the concept of a tiling axiomatically and prove that tilings exist; then we will deduce the existence of the retractions.

The simplest example of a tiling is where $X$ is the upper half plane and $\Gamma=$ $\mathrm{SL}(2, \mathbb{Z})$. Here $\bar{X}_{P}$ is an open horocyclic neighborhood of the rational boundary point which the maximal parabolic $P$ fixes; these are all $\Gamma$-translates of each other. The set $\bar{X}_{G}=X_{0}$ is the complement of all these open horoballs; see Figure 1. The situation in the general $\mathbb{Q}$-rank 1 case is not very different. 


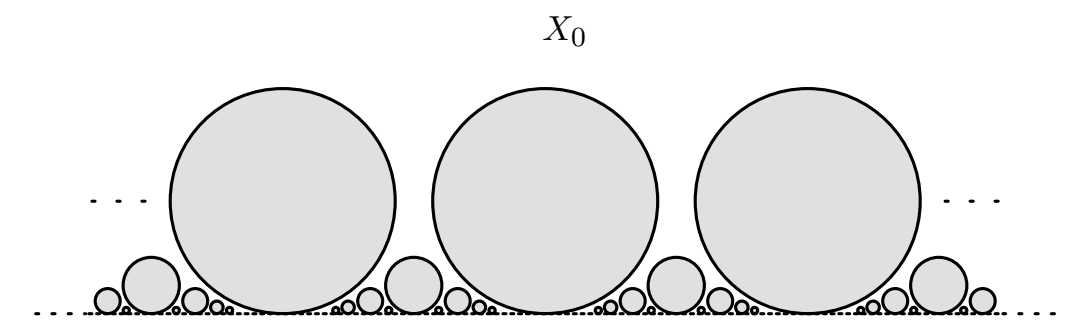

Figure 1.

The tiling for the upper half plane, $\Gamma=\mathrm{SL}(2, \mathbb{Z})$

In higher rank, however, the geometry of the tilings is more interesting; the $\mathbb{Q}$-rank 2 case is represented in Figure 2. The intersection of $\bar{X}_{P}$ with each $A_{P}$ fiber is an open acute cone defined by the roots of $P$ - the strictly dominant cone; the retraction maps $\bar{X}_{P}$ onto $\partial^{P} X_{0}$ by collapsing these cones. On the other hand, the intersection of $X_{0}$ with each $A_{P}$ fiber is not contained in the negative of the dominant cone, but rather the negative of the closed obtuse cone corresponding to the dual basis (the codominant cone). This situation is forced upon us by the requirements we have placed on $r$ and illustrates the beginning of the difficulties mentioned above.

If one restricts to the interior $X$ and requires that the faces of $X_{0}$ lie near infinity, the existence of such tilings is not new. They occur in the theory of the trace formula and were constructed by Arthur [1] in the adelic case following Langlands [31]; a construction within the axiomatic framework considered by Langlands (which includes the case of arithmetic groups) was given by Osborne and Warner [39].

In this paper we begin (after some background material in $\S 1$ ) with an axiomatic definition of tilings in $\S 2$ and deduce some basic properties. The actual construction proceeds in three steps, occuring in $\S \S 3-5$ respectively, which mirror those in the construction of $\bar{X}$ :

(1) Construct a model tiling of $\bar{A}_{P}, \coprod_{R \supseteq P}\left\langle\bar{A}_{P}\right\rangle_{R}$. This is an extension of Langlands's geometric partition of $A_{P}$.

(2) Shift this tiling of $\bar{A}_{P}$ by a parameter $b_{P} \in A_{P}$ and use the decomposition $X(P) \cong \bar{A}_{P} \times e(P)$ to induce a tiling $X(P)=\coprod_{R \supseteq P} X(P)_{R} \equiv \coprod_{R \supseteq P} b_{P}$. $\left\langle\bar{A}_{P}\right\rangle_{R} \times e(P)$. We call this the tiling associated to $P$.

(3) Intersect the tilings associated to all parabolics, that is, define $\bar{X}_{R} \equiv$ $\bigcap_{P \subseteq R} X(P)_{R}$. If the parameters $b_{P}$ are sufficiently large and suitably 


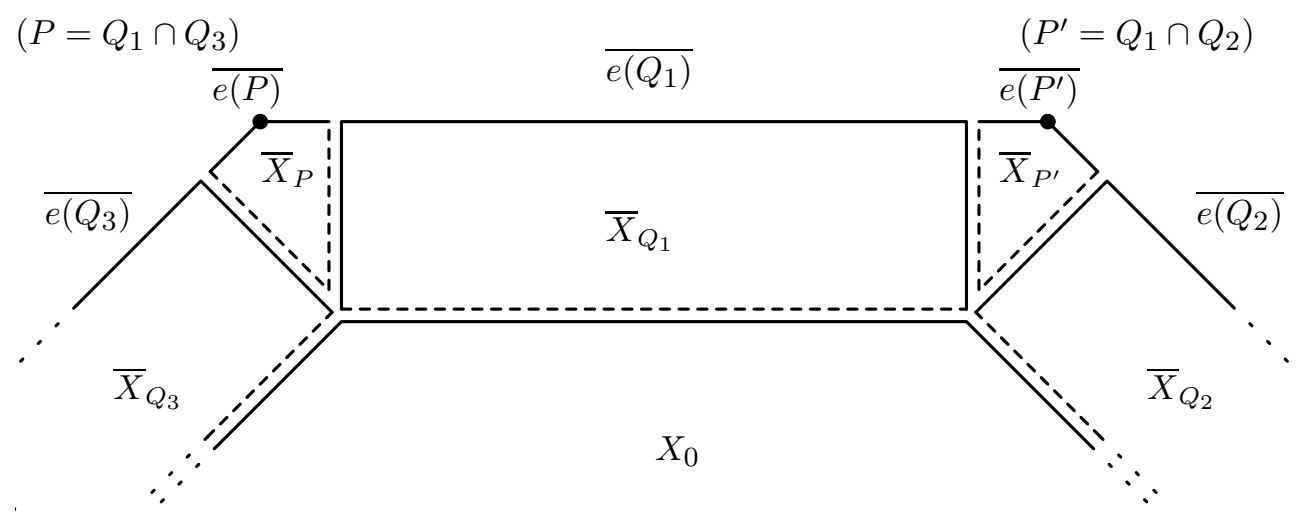

Figure 2 .

The tiling in the $\mathbb{Q}$-rank 2 case $^{1}$

(The tiles been separated slightly to indicate the boundaries.)

$\Gamma$-invariant and compatible, this will be a $\Gamma$-invariant tiling of $\bar{X}$.

Tilings have many applications other than for the trace formula - some requiring the extension to $\bar{X}$ and some concerned with $X_{0}$ collapsing into the interior. Here are a few examples we will consider in this paper.

\section{Finite energy retractions}

We have already indicated that a tiling may be used to construct a deformation retraction of $\Gamma \backslash X$ with compact image; this is done in $\S 6$. The explicit nature of this map enables us to determine in $\S 7$ precisely when it has finite energy: for irreducible $\Gamma \backslash X$ the retraction has finite energy except in a few low rank cases; in fact, if we simply require a weaker condition of almost finite energy, the only exception is when $G=\mathrm{SL}(2, \mathbb{R})$. Thus any map of $\Gamma \backslash X$ to a Riemannian manifold $N$ may be deformed to one that factors through the compact set $\Gamma \backslash X_{0}$ and thus has finite energy (aside from the above exceptions).

As Borel has indicated, this result has important applications in the harmonic map approach to geometric rigidity. The motivation is that when $\Gamma \backslash X$ is compact and $N$ has nonpositive curvature, a map $\Gamma \backslash X \rightarrow N$ may be deformed to a harmonic map. Then results proved by Corlette [17] (for real rank one) and independently by Mok, Siu, Yeung [38] and Jost and Yau [29] (for higher rank) show that in most cases such harmonic maps are actually totally geodesic embeddings. The obstruction to carrying out such an argument when $\Gamma \backslash X$ is noncompact has been

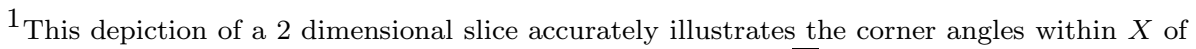
the tiles. (The metric and hence the angles are not defined on $\bar{X} \backslash X$.) The picture does not represent the analytic structure correctly, however; in particular, the lower boundary of $\bar{X}_{Q_{1}}$ does not, if naturally extended, meet $e\left(Q_{2}\right)$ and $e\left(Q_{3}\right)$ as one might think. For other depictions, see Figures 3 and 4 .
} 
the existence of a finite energy map which could then be deformed into a harmonic map. In real rank one, Corlette uses the well-known structure of the cusps [19] to show a finite energy retraction exists; our work handles the general case. (Indeed, a recent preprint of Jost and $\mathrm{Li}[28]$ shows that even our condition of almost finite energy is sufficient for the construction of a harmonic map.)

In the compact case, the above results together with the analogous nonarchimedean version due to Gromov and Schoen [25] yield a new proof of Margulis's theorem on arithmeticity of lattices; in the real rank one case it extends Margulis's work. Unfortunately, the present paper cannot be used for this purpose since we already assume $\Gamma$ is arithmetic and rely on the reduction theory for arithmetic groups [9]. On the other hand, since by Margulis's theorem [35] the only nonarithmetic irreducible lattices occur in groups of real rank 1 and the cusps of such $\Gamma \backslash X$ are understood by [19], our results hold true for arbitrary lattices. See also [37] and [30] for other work on the application of harmonic maps to geometric rigidity, as well as [7], [8], [16], and [18] where results on geometric rigidity are obtained by other means.

\section{Diffeomorphisms}

Another application of tilings (and our initial motivation) is to construct a canonical piecewise analytic diffeomorphism $\Gamma \backslash \bar{X} \rightarrow \Gamma \backslash X_{0}$; this is done in $\S 6$ along with the retraction. Thus $\Gamma \backslash X_{0}$ is actually a natural realization of the Borel-Serre compactification within $\Gamma \backslash X$. This diffeomorphism is the first step in our approach to the conjecture of Rapoport [41] and Goresky and MacPherson [21] on the intersection cohomology of the reductive Borel-Serre compactification, which we will discuss elsewhere.

\section{Neighborhoods}

The difficulty of finding natural neighborhoods of the closed boundary faces $\overline{e(P)}$ of $\Gamma \backslash \bar{X}$ was mentioned previously. We will see in $\S 8$ that tilings can be applied to yield an explicit cofinal family of $(\Gamma \cap P)$-invariant neighborhoods of $\overline{e(P)}$ with piecewise analytic boundaries. In the hermitian symmetric case these reduce to the "adapted cores" of $[43, \S 4]$ which played a crucial role in Saper and Stern's proof of Zucker's conjecture.

\section{Collapsing $\Gamma \backslash \bar{X}$}

A particular tiling is determined by specifying how far the maximal faces of $X_{0}$ lie from the maximal faces of $\bar{X}$; this is measured by a parameter $\mathbf{b}=\left(b_{Q}\right)_{Q}$, where $b_{Q} \in A_{Q}$ and $Q$ runs over maximal parabolic $\mathbb{Q}$-subgroups. (Of course for the tiling to be $\Gamma$-invariant, $\mathbf{b}$ must satisfy an appropriate $\Gamma$-invariance condition.) As the $b_{Q}$ tend to $\infty$, the tiling degenerates by having $\Gamma \backslash X_{0}$ expand to fill up the entire space. However, it is also of interest to study degenerations of tilings in the opposite sense, when the boundary faces of $\Gamma \backslash X_{0}$ collapse inward. In Figure 1, this means that the horocycles expand until they touch as pictured in Serre [45]. 
As a start toward such a study, we consider in $\S 9$ the space of all parameters $\mathbf{b}$ for which tilings exist. For such a parameter (with $\mathbf{b}$ not necessarily large) it is not clear that the tiling is obtained as in step (3) of the construction above. Fortunately this step is not used when working with tilings (at least not in the applications above); instead one uses the existence of the tiling and the property that in certain cylindrical sets, the tiling agrees with a tiling associated to a parabolic (as in step (2)). We will show that this property holds for all tilings. We also give a criterion for a parameter to admit a tiling; from this we find that the space of such parameters is open and invariant under the action of the dominant cone in a maximal $\mathbb{Q}$-split torus.

Further degeneration is also conceivable, in which the boundary faces of $\Gamma \backslash X_{0}$ do not merely touch, but begin to flatten out against each other until $\Gamma \backslash X_{0}$ collapes onto a lower dimensional subspace. We do not consider this in the current paper, but it is reasonable to speculate that such a process would yield retractions generalizing those in the work of Ash [2] (the "well-rounded retract", special cases of which were constructed previously by Mendoza [36] and Soulé [46]) and MacPherson and McConnell [34]. Such retractions have applications to the cohomology of arithmetic groups and the theory of exact fundamental domains. In this connection, we note the recent paper of Ash and McConnell [3] in which the deformation retraction onto the well-rounded retract is extended to the Borel-Serre compactification.

The present paper is set in the context of symmetric spaces and semisimple groups. However all the results generalize without difficulty to the case of homogeneous spaces of type $S-\mathbb{Q}[12, \S 2.3]$; we leave this to the reader.

We finally note that other interesting decompositions of $X$ are possible. The constructions of Harder [27, §1.2] may be used to construct a partition of $X$ which is different from the tilings considered here. This has been carried out by Grayson [22], [23], who uses an approach to reduction theory via semistability (see [5]) and finds an explicit and canonical partition and retraction. Grayson was also motivated by [26] and Stuhler [47], [48]. Also the recent paper of Leuzinger [33] constructs an exhaustion of $X$ by regions analogous to our $X_{0}$ by geometric means. ${ }^{2}$

My indebtedness to the published work of many mathematicians is already apparent from the above. I would like to express my deep thanks to Armand Borel for his interest and encouragement of this work. I would also like to thank Shing-Tung Yau who first asked me whether finite energy retractions with compact image exist, and conjectured that they did for rank $>2$. Also conversations with Bill Casselman, Pat Eberlein, David Morrison, Jürgen Rohlfs, Rick Schoen, and Joachim Schwermer were very helpful. Mark Goresky made several suggestions

\footnotetext{
2 In [33] the regions are denoted $X(s)$, where $s$ is a sufficiently large real parameter. They correspond here to the central tiles $X_{0}$ for a family of tilings whose parameters are $t \cdot \mathbf{b}$, where $\mathbf{b}$ is fixed and $t$ belongs to a strictly dominant 1-parameter subgroup of a maximal $\mathbb{Q}$-split torus. (See $\S 6$ where such regions are denoted $X_{0, t}$.)
} 
for revisions to a very early version of this paper [42] which were greatly appreciated. Finally I would like to thank Mark McConnell and especially the referee for extremely valuable suggestions regarding the exposition and organization.

\section{Background}

In order to set notation, we briefly recall without proofs some standard facts regarding algebraic groups over $\mathbb{Q}$, the geodesic action, and the Borel-Serre compactification.

Algebraic groups

Let $G$ be the identity component of the real points of a semisimple algebraic group defined over $\mathbb{Q}$ and let $\Gamma \subset G_{\mathbb{Q}}$ be an arithmetic subgroup. Lie algebras will be denoted by the corresponding lower case gothic letter, e.g., $\mathfrak{g}$. We denote by $\mathcal{P}$ the set of parabolic $\mathbb{Q}$-subgroups of $G$ (including $G$ itself) and by $\mathcal{P}_{1}$ the set of maximal proper parabolic $\mathbb{Q}$-subgroups. By a parabolic (resp. maximal parabolic) we always mean an element of $\mathcal{P}$ (resp. $\left.\mathcal{P}_{1}\right)$. We will reserve the letter $Q$ to denote a maximal parabolic. If $R, S \in \mathcal{P}$, we denote by $R \vee S$ the smallest parabolic containing $R \cup S$.

For a parabolic $P$, let $N_{P}$ be the unipotent radical of $P$ and let $A_{P}$ be the identity component of the maximal $\mathbb{Q}$-split torus in the center of $P / N_{P}$. The parabolic rank of $P\left(\operatorname{denoted} \mathcal{P}\right.$-rank $P$ ) is $\operatorname{dim} A_{P}$. Conjugation by $g \in G_{\mathbb{Q}}$ allows us to canonically identify $A_{P}$ and $A_{g_{P}}[12, \S 4.2]$. (We denote conjugation as ${ }^{g} P=P^{g^{-1}}=g P g^{-1}$, for example.) Let ${ }^{0} P \subseteq P$ be the subgroup $\{p \in P \mid$ $\left|p^{\chi}\right|=1$ for all $\left.\chi \in \operatorname{Mor}_{\mathbb{Q}}\left(P, \mathrm{GL}_{1}\right)\right\}$ as in $[12, \S 1.1] ;{ }^{0} P$ contains $N_{P}$ as well as any compact or arithmetic subgroup of $P[12, \S 1.2]$. We write $\Gamma_{P} \equiv \Gamma \cap P \subset{ }^{0} P$.

Let $\Delta_{P}$ denote the simple "roots" of the adjoint action of (a lift of) $A_{P}$ on $\mathfrak{n}_{P}$; we view elements of $\Delta_{P}$ both as characters of $A_{P}$ and as elements of $\mathfrak{a}_{P}^{*}$. Let $\widehat{\Delta}_{P}=\left\{\beta_{\alpha}\right\}_{\alpha \in \Delta_{P}}$ be the dual basis of $\mathfrak{a}_{P}^{*}$ relative to a Weyl group invariant inner product. For a parabolic $R \supseteq P$, the group $A_{R}$ may be canonically identified with a subgroup of $A_{P}[12, \S 3.11]$; let $\Delta_{P}^{R} \subseteq \Delta_{P}$ denote those roots restricting to 1 on $A_{R}$. The set $\Delta_{P}^{R}$ is called the type of $R$ (relative to $P$ ). Then $A_{R}=\bigcap_{\alpha \in \Delta_{P}^{R}} \operatorname{Ker} \alpha$ and we define $A_{P}^{R}=\bigcap_{\gamma \notin \Delta_{P}^{R}} \operatorname{Ker} \beta_{\gamma}$. This yields an orthogonal decomposition

$$
A_{P} \cong A_{R} \times A_{P}^{R}
$$

we denote the corresponding decomposition of $a \in A_{P}$ by $a_{R} a^{R}$, and similarly for elements of $\mathfrak{a}_{P}$ or $\mathfrak{a}_{P}^{*}$. For $Q$ a maximal parabolic it is convenient to denote by $\beta_{Q}$ the unique element of $\widehat{\Delta}_{Q}$. Thus we have $\widehat{\Delta}_{R}=\left\{\beta_{Q}\right\}_{Q \supseteq R}$, while if $P \subseteq R$, the dual basis to $\Delta_{P}^{R}$ of $\mathfrak{a}_{P}^{R^{*}}$ is $\widehat{\Delta}_{P}^{R}=\left\{\beta_{Q}^{R}\right\}_{Q \supseteq P, Q \nsupseteq R}$.

We omit the subscript $P$ in all these notations when $P$ is minimal, and similarly we omit the superscript when it is $G$. Sometimes (particularly in $\S 3$ ) we use the type of $R$ as a subscript or superscript, instead of $R$ itself. 
Let $(S, \Delta)$ be any pair $\left(A_{P}^{R}, \Delta_{P}^{R}\right)$. We call $a \in S$ dominant (with respect to $\Delta$ ) if $a^{\alpha} \geq 1$ for all $\alpha \in \Delta$; $a$ is codominant (with respect to $\Delta$ ) if $a^{\beta_{\alpha}} \geq 1$ for all $\alpha \in \Delta$. If these inequalities are strict for all $\alpha$, we say $a$ is strictly dominant (resp. strictly codominant). The strictly dominant cone is denoted $S(1)$ and in general for $b \in S$, set $S(b)=b \cdot S(1)$. We will also transfer this terminology to $\mathfrak{s}$ or (by using the inner product) to $\mathfrak{s}^{*}$. The dominant functionals in $\mathfrak{s}^{*}$ form a convex cone generated by $\widehat{\Delta}$, while the codominant functionals form the dual convex cone generated by $\Delta$.

Geodesic action

Let $X=G / K$ be a Riemannian symmetric space of noncompact type, where $K \subset G$ is a maximal compact subgroup stabilizing a fixed basepoint $x \in X . P$ acts transitively on $X$, so $z \in X$ may be expressed as $z=p x$ with $p \in P$. The geodesic action of $A_{P}[12, \S 3.2]$ is defined by

$$
a \mathbf{o} z=p a_{x} x \quad\left(a \in A_{P}, z \in X\right),
$$

where $a_{x} \in A_{P, x}$ is the unique lift of $a \in A_{P}$ to $P$ stable under the Cartan involution associated to $x[12, \S \S 1.6,1.8]$. The geodesic action commutes with the usual action of $P[12, \S 3.2]$, and for $P \subseteq R \in \mathcal{P}$, the geodesic action of $A_{R}$ is the restriction of the geodesic action of $A_{P}[12, \S 3.11]$.

Let $A_{P} \times{ }^{0} P$ act on $X$ by the product of the geodesic action and the usual action for ${ }^{0} P$. Then there is an analytic isomorphism

$$
\left(a_{P}, q_{P}\right): X \stackrel{\sim}{\longrightarrow} A_{P} \times e(P)
$$

of $\left(A_{P} \times{ }^{0} P\right)$-homogeneous spaces $[12, \S 3.8]$, where $e(P)=A_{P} \backslash X$ is the quotient under the geodesic action. We normalize (1.2) such that $a_{P}(x)=1$, where $x$ is our fixed basepoint. In other words, $X$ is a trivial principal $A_{P}$-bundle with canonical cross-sections given by orbits of ${ }^{0} P$. We will often treat (1.2) as an identification with the parabolic being clear from the context.

\section{Borel-Serre compactification}

The bordification $\bar{X}$ may now be defined by a three step procedure:

(1) The roots $\Delta_{P}$ induce an isomorphism $A_{P} \stackrel{\sim}{\rightarrow}\left(\mathbb{R}^{>0}\right)^{\Delta_{P}}$ by $a \mapsto\left(a^{\alpha}\right)_{\alpha \in \Delta_{P}}$. Thus we may naturally embed $A_{P}$ into a semigroup $\bar{A}_{P} \cong\left(\mathbb{R}^{>0} \cup\{\infty\}\right)^{\Delta_{P}}$ $[12, \S 4.4]$; the inverses of the root coordinates on $\bar{A}_{P}$ yield a real analytic structure.

(2) A partial bordification $X(P)$ (the corner associated to $P$ ) is obtained as $X(P) \equiv \bar{A}_{P} \times_{A_{P}} X$, or equivalently by extending $(1.2)$ to $\left(a_{P}, q_{P}\right): X(P) \stackrel{\sim}{\rightarrow}$ $\bar{A}_{P} \times e(P)[12, \S 5.1]$. We identify $e(P)$ with $\{\infty\}^{\Delta_{P}} \times e(P)$.

(3) For parabolics $P \subseteq R$, there is a natural inclusion of $X(R)$ into $X(P)$ as an open subset $[12, \S 5.3]$. One defines $\bar{X} \equiv \bigcup_{P \in \mathcal{P}} X(P)$ to have the unique 
structure of analytic manifold with corners so that each $X(P)$ is an open submanifold with corners $[12, \S 7.1]$. Note that $\bar{X}$ may be decomposed as $\bigsqcup_{P \in \mathcal{P}} e(P)$, where $e(G)=X$.

The action of $G_{\mathbb{Q}}$ on $X$ extends to $\bar{X}[12, \S 7.6]$. $\Gamma$ acts properly on $\bar{X}$ and $\Gamma \backslash \bar{X}$ is compact $[12, \S 9.3]$; we denote the quotient map $\pi: \bar{X} \rightarrow \Gamma \backslash \bar{X}$.

We denote topological closure by $\operatorname{cl}(\cdot)$, however in the case of $\operatorname{cl}(e(P)) \subseteq \bar{X}$, one writes $\overline{e(P)}$ to emphasize that this space may also be constructed analogously to $\bar{X}$ by letting $P$ take the role of $G[12, \S 7.3(\mathrm{i})]$. (One must work in the wider context of homogeneous spaces of type $S-\mathbb{Q}[12, \S 2.3]$.) The association $P \mapsto \overline{e(P)}$ is an inclusion preserving, $G_{\mathbb{Q}^{-}}$-equivariant bijection between $\mathcal{P}$ and the closed boundary faces of $\bar{X}[12, \S 7.4]$.

\section{Tilings of $\bar{X}$}

In this section we define the concept of tilings and prove some of their basic properties. A construction of tilings will be given in $\S \S 3-5$.

Definition 2.1. A tiling of $\bar{X}$ is a cover $\bar{X}=\coprod_{P \in \mathcal{P}} \bar{X}_{P}$ by disjoint sets (called tiles), having the following properties:

(i) The central tile $X_{0} \equiv \bar{X}_{G}$ is a closed, codimension 0 submanifold with corners contained in $X$. Its closed boundary faces $\left\{\partial^{P} X_{0}\right\}$ may be indexed by $P \in \mathcal{P}$ so that $P \mapsto \partial^{P} X_{0}$ is an inclusion preserving bijection.

(ii) Each boundary face $\partial^{P} X_{0}$ lies in a canonical cross-section $\left\{b_{P}\right\} \times e(P)$.

(iii) Each tile $\bar{X}_{P}$ is obtained from $\partial^{P} X_{0}$ by flowing out under the geodesic action of the cone $\bar{A}_{P}(1)$, that is, $\bar{X}_{P}=\bar{A}_{P}(1)$ o $\partial^{P} X_{0}$.

The tiling is called $\Gamma$-invariant if $\gamma \cdot \bar{X}_{P}=\bar{X}_{\gamma P}$ for all $\gamma \in \Gamma$ and $P \in \mathcal{P}$.

Remark. We similarly define the concept of a tiling of $e(P), X(P), A_{P}$, etc. In the first case, we restrict the indexing set to parabolics contained in $P$; in the latter two cases, we restrict the indexing set to parabolics containing $P$.

The following basic properties of a tiling are analogues of properties of the decomposition $\bar{X}=\bigsqcup_{P \in \mathcal{P}} e(P)$ of $[12]$.

Proposition 2.2. A tiling $\bar{X}=\coprod_{P \in \mathcal{P}} \bar{X}_{P}$ satisfies the following properties:

(i) Each tile $\bar{X}_{P}$ is a codimension 0 submanifold with corners.

(ii) The closures of any two tiles are either disjoint or intersect in a common closed boundary face. More precisely, for $P, P^{\prime} \in \mathcal{P}$,

$$
\operatorname{cl}\left(\bar{X}_{P}\right) \cap \operatorname{cl}\left(\bar{X}_{P^{\prime}}\right)= \begin{cases}\operatorname{cl}\left(\bar{A}_{P \vee P^{\prime}}(1)\right) \mathbf{o} \partial^{P \cap P^{\prime}} X_{0} & \text { if } P \cap P^{\prime} \in \mathcal{P}, \\ \emptyset & \text { otherwise. }\end{cases}
$$


If the tiling is $\Gamma$-invariant, then furthermore:

(iii) $\Gamma \backslash X_{0}$ is compact.

(iv) For all $P \in \mathcal{P}$, the natural projection $\Gamma_{P} \backslash \operatorname{cl}\left(\bar{X}_{P}\right)=\bar{A}_{P}(1) \mathbf{o} \Gamma_{P} \backslash \partial^{P} X_{0} \rightarrow$ $\pi\left(\operatorname{cl}\left(\bar{X}_{P}\right)\right)$ is a homeomorphism.

Proof. Part (i) follows easily from the definitions. For (ii) note that by Definition 2.1(iii), $\bar{X}_{P}=\bar{A}_{P}(1) \mathbf{o} \partial^{P} X_{0}$, and thus after taking closure, $\operatorname{cl}\left(\bar{X}_{P}\right)=$ $\operatorname{cl}\left(\bar{A}_{P}(1)\right) \mathbf{o} \partial^{P} X_{0}$. However $\operatorname{cl}\left(\bar{A}_{P}(1)\right)=\coprod_{R \supseteq P} \bar{A}_{R}(1)$. Furthermore, $\partial^{P} X_{0} \subseteq$ $\partial^{R} X_{0}$ for $R \supseteq P$ by Definition 2.1(i). Thus we may compute

Hence for $P, P^{\prime} \in \mathcal{P}$,

$$
\operatorname{cl}\left(\bar{X}_{P}\right)=\coprod_{R \supseteq P} \bar{A}_{R}(1) \mathbf{o} \partial^{P} X_{0} \subseteq \coprod_{R \supseteq P} \bar{X}_{R} .
$$

$$
\begin{aligned}
\operatorname{cl}\left(\bar{X}_{P}\right) \cap \operatorname{cl}\left(\bar{X}_{P^{\prime}}\right) & =\coprod_{R \supseteq P \cup P^{\prime}}\left(\bar{A}_{R}(1) \mathbf{o} \partial^{P} X_{0}\right) \cap\left(\bar{A}_{R}(1) \mathbf{o} \partial^{P^{\prime}} X_{0}\right) \\
& =\coprod_{R \supseteq P \cup P^{\prime}} \bar{A}_{R}(1) \mathbf{o}\left(\partial^{P} X_{0} \cap \partial^{P^{\prime}} X_{0}\right) .
\end{aligned}
$$

By Definition 2.1(i), this last expression is empty unless $P \cap P^{\prime} \in \mathcal{P}$, in which case it is equal to

$$
\coprod_{R \supseteq P \cup P^{\prime}} \bar{A}_{R}(1) \mathbf{o} \partial^{P \cap P^{\prime}} X_{0}=\operatorname{cl}\left(\bar{A}_{P \vee P^{\prime}}(1)\right) \mathbf{o} \partial^{P \cap P^{\prime}} X_{0} .
$$

This finishes the proof of (ii).

Now assume the tiling is $\Gamma$-invariant. Since $\Gamma \backslash X_{0}$ is a closed subset of the compact space $\Gamma \backslash \bar{X}$, (iii) is clear. Finally for (iv), let $x, y \in \operatorname{cl}\left(\bar{X}_{P}\right)$ and say $\gamma x=y$ for $\gamma \in \Gamma$. Then $\operatorname{cl}\left(\bar{X}_{P}\right) \cap \operatorname{cl}\left(\bar{X}_{\gamma P}\right)=\operatorname{cl}\left(\bar{X}_{P}\right) \cap \gamma \cdot \operatorname{cl}\left(\bar{X}_{P}\right) \neq \emptyset$. By (ii), $P \cap{ }^{\gamma} P \in \mathcal{P}$ which implies $P={ }^{\gamma} P$ and thus $\gamma \in \Gamma_{P}$.

Definition 2.6. The parameter of a tiling is the collection $\mathbf{b}=\left(b_{Q}\right)_{Q \in \mathcal{P}_{1}}$ from Definition 2.1. The space of all parameters is denoted $\mathcal{B} \equiv \prod_{Q \in \mathcal{P}_{1}} A_{Q} \cong\left(\mathbb{R}^{>0}\right)^{\mathcal{P}_{1}}$.

Remark. (1) Note that we only include $b_{Q}$ in $\mathbf{b}$ for $Q$ maximal since the canonical cross-section $\left\{b_{P}\right\} \times e(P)$ is determined as the intersection of the canonical crosssections $\left\{b_{Q}\right\} \times e(Q)$ for $Q \supseteq P$. Also note that our assignment of parameters to a tiling depends on our fixed basepoint.

(2) When dealing with families of tilings, it will be helpful to use the action of the maximal torus $A$ on $\mathcal{B}$ given by $t \cdot\left(b_{Q}\right)_{Q \in \mathcal{P}_{1}} \equiv\left(t_{Q} \cdot b_{Q}\right)_{Q \in \mathcal{P}_{1}}$.

A tiling is uniquely determined by its parameter $\mathbf{b} \in \mathcal{B}$ (for later use, we in fact prove a more general result): 
Proposition 2.4. Let $\mathbf{b} \in \mathcal{B}$ be a parameter and $\bar{M} \subseteq \bar{X}$ an open subset. There is at most one decomposition $\bar{M}=\coprod_{R \in \mathcal{P}} \bar{M}_{R}$ for which $\bar{M}_{R} \subseteq X(R)$ is $\operatorname{cl}\left(\bar{A}_{R}(1)\right)$ invariant and satisfies

$$
\bar{M}_{R}=\left(\bar{A}_{R}\left(b_{R}\right) \times q_{R}\left(\bar{M}_{R}\right)\right) \cap \bar{M} .
$$

Proof. Clearly $\bar{M}_{P} \cap \overline{e(R)}=\emptyset$ unless $P \subseteq R$, in which case it equals $q_{R}\left(\bar{M}_{P}\right)$. Thus $\bar{M} \cap \overline{e(R)}=\coprod_{P \in \mathcal{P}} \bar{M}_{P} \cap \overline{e(R)}=\coprod_{P \subseteq R} q_{R}\left(\bar{M}_{P}\right)$, and consequently

$$
q_{R}\left(\bar{M}_{R}\right)=(\bar{M} \cap \overline{e(R)}) \backslash \bigcup_{P \subsetneq R} q_{R}\left(\bar{M}_{P}\right) .
$$

Equations (2.2) and (2.3) determine $\bar{M}_{R}$ directly for $R$ minimal, and by recursion on parabolic rank in general.

Corollary 2.5. At most one tiling of $\bar{X}$ exists for a given parameter $\mathbf{b} \in \mathcal{B}$.

Proof. Apply the proposition with $\bar{M}=\bar{X}$ and $\bar{M}_{R}=\bar{X}_{R}$; the required hypotheses follow from Definition 2.1(ii)(iii).

We now consider the naturality of tilings and their parameters under automorphisms of $G$ and in particular, how $\Gamma$-invariance of a tiling is reflected in its parameter. Let $\phi: G \rightarrow G$ be an automorphism defined over $\mathbb{Q}$. Then $\phi$ acts on $X$, viewed as the space of maximal compact subgroups of $G$. Define an action of $\phi$ on $\mathcal{B}$ by

$$
\phi \cdot\left(b_{Q}\right)_{Q \in \mathcal{P}_{1}}=\left(c(\phi, Q) \phi\left(b_{Q}\right)\right)_{\phi(Q) \in \mathcal{P}_{1}}
$$

where we set

$$
c(\phi, P)=a_{\phi(P)}(\phi \cdot x) .
$$

(We have to be careful since $\phi$ may move the fixed basepoint $x$.) When $\phi$ is the inner automorphism induced by an element $g \in G_{\mathbb{Q}}$, we simply write $g \cdot \mathbf{b}$ and $c(g, P)$.

Proposition 2.6. $\phi$ induces an automorphism of $\mathcal{B}$. If $\left\{\bar{X}_{R}\right\}_{R \in \mathcal{P}}$ is a tiling (resp. $\Gamma$-invariant tiling) with parameter $\mathbf{b}$, then $\left\{\phi \cdot \bar{X}_{R}\right\}_{\phi(R) \in \mathcal{P}}$ is a tiling (resp. $\phi(\Gamma)$-invariant tiling) with parameter $\phi \cdot \mathbf{b}$.

Proof. The action of $\phi$ transforms a canonical cross-section $\{a\} \times e(P)$ to a canonical cross-section $\{c(\phi, P) \phi(a)\} \times e(\phi(P))$. Now use the definition and Corollary 2.5 .

Corollary 2.7. A tiling is $\Gamma$-invariant if and only if its parameter $\mathbf{b}$ satisfies

$$
b_{\gamma_{Q}}=c(\gamma, Q) \cdot b_{Q}
$$


for all $\gamma \in \Gamma$ and $Q \in \mathcal{P}_{1}$.

Let $\mathcal{B}^{\Gamma} \subset \mathcal{B}$ be the subspace of parameters satisfying (2.4); we call these the $\Gamma$-invariant parameters. Such a parameter is determined by a choice of $b_{Q}$ for each $\Gamma$-conjugacy class of maximal parabolic subgroups:

Proposition 2.8. Let $\mathcal{R}_{1}(\Gamma)$ be a set of representatives of $\Gamma$-conjugacy classes of maximal parabolics. The natural map $\mathcal{B}^{\Gamma} \rightarrow \prod_{Q \in \mathcal{R}_{1}(\Gamma)} A_{Q} \cong\left(\mathbb{R}^{>0}\right)^{\mathcal{R}_{1}(\Gamma)}$ is a bijection.

Proof. The map is clearly injective; we need to show it is surjective. Consider $\left(b_{Q}\right)_{Q \in \mathcal{R}_{1}(\Gamma)}$. For $Q \in \mathcal{R}_{1}(\Gamma)$ and $\gamma^{\prime}$ running over a set of representatives of $\Gamma / \Gamma_{Q}$, set $b_{\gamma^{\prime} Q}=c\left(\gamma^{\prime}, Q\right) \cdot b_{Q}$. Note that if $\gamma \in \Gamma$ is such that ${ }^{\gamma} Q=\gamma^{\prime} Q$, then $\gamma^{\prime-1} \gamma \in \Gamma_{Q} \subseteq{ }^{0} Q$. Thus $c(\gamma, Q)=c\left(\gamma^{\prime}, Q\right)$ and so $b_{\gamma_{Q}}=c(\gamma, Q) \cdot b_{Q}$ as well.

\section{Construction of tilings, I: Tilings of $\bar{A}_{P}$}

In this section and the following two we will demonstrate that tilings of $\bar{X}$ with parameter $\mathbf{b}$ exist, provided $\mathbf{b}$ is sufficiently large and $\Gamma$-invariant. Our construction has three parts, mirroring the three steps in the construction of the Borel-Serre bordification $\bar{X}$. In this first part we begin by recalling the tiling of $A_{P}$ (or equivalently $\mathfrak{a}_{P}$ ) due to Langlands, and show it extends to an analytic tiling of $\bar{A}_{P}$ (Corollary 3.8); it is necessary and actually simpler to work in a more general context.

Let $(V, \Delta)$ be a pair consisting of a finite dimensional real vector space with inner product $(\cdot, \cdot)$ and a basis $\Delta$ for the dual inner product space $V^{*}$. We assume that

$$
\left(\alpha, \alpha^{\prime}\right) \leq 0 \quad\left(\alpha \neq \alpha^{\prime} \in \Delta\right) .
$$

For example, $(\mathfrak{a}, \Delta)$ satisfies (3.1) since $\Delta$ is the basis of the $\mathbb{Q}$-root system of $G$. More generally, we may consider $\left(\mathfrak{a}_{P}^{R}, \Delta_{P}^{R}\right)$ for parabolics $P \subseteq R$ :

Lemma 3.1 ([13, IV, 6.4)]. For $I \subseteq \Delta$ the pairs $\left(V_{I}, \Delta_{I}\right) \equiv\left(\bigcap_{\alpha \in I} \operatorname{Ker} \alpha,\left\{\gamma_{I}\right\}_{\gamma \notin I}\right)$ and $\left(V^{I}, \Delta^{I}\right) \equiv\left(\bigcap_{\gamma \notin I} \operatorname{Ker} \beta_{\gamma}, I\right)$ satisfy the hypothesis (3.1) of this section. The respective dual bases are $\widehat{\Delta}_{I} \equiv\left\{\beta_{\gamma}\right\}_{\gamma \notin I}$ and $\widehat{\Delta}^{I} \equiv\left\{\beta_{\alpha}^{I}\right\}_{\alpha \in I}$.

Note that we are applying to $V$ the notation introduced in (1.1) and following, except that instead of using parabolics as subscripts and superscripts, we use subsets of $\Delta$ (the type). Thus we have an orthogonal decomposition

$$
V=V_{I} \oplus V^{I}
$$


for any $I \subseteq \Delta$.

We will often use the following facts regarding dominance and codominance and their behavior under this decomposition:

Lemma 3.2. The dominant cone is contained within the codominant cone. The various $\beta_{\alpha}$ 's are mutually acute (or orthogonal). If $\lambda \in V^{*}$ is (strictly) dominant (resp. codominant) with respect to $\Delta$, then $\lambda_{I} \in V_{I}^{*}$ is (strictly) dominant (resp. codominant) with respect to $\Delta_{I}$. If $\lambda$ is (strictly) dominant with respect to $\Delta$, then $\lambda^{I} \in V^{I *}$ is (strictly) dominant with respect to $\Delta^{I}$.

Proof. The first assertion is [13, IV, §6.2] and the second follows from the first. The rest is obvious except for the (strict) dominance assertion for $\lambda_{I}$, which follows from [13, IV, §6.5(2)] (and the following remark).

Remark 3.3. (i) It is not the case that $\lambda \in V^{*}$ codominant implies that $\lambda^{I} \in V^{I *}$ is codominant with respect to $\Delta^{I}$. In fact, if $\gamma \notin I$, then $\gamma^{I}=-\sum_{\alpha \in I} c_{\alpha} \beta_{\alpha}^{I}$, where $c_{\alpha}=-(\gamma, \alpha) \geq 0$. Thus $\gamma^{I}$ is antidominant with respect to $\Delta^{I}$.

(ii) By the lemma, the notion of dominance or codominance for a functional in $V_{I}^{*} \subseteq V^{*}$ is the same with respect to $\Delta_{I}$ or with respect to $\Delta$. Likewise, a functional in $V^{I *}$ is codominant with respect to $\Delta^{I}$ if and only if it is codominant with respect to $\Delta$. Thus in these cases, we shall not mention the basis.

Define

$$
\langle V\rangle_{\Delta}=\left\{v \in V \mid \beta_{\alpha}(v) \leq 0 \text { for all } \beta_{\alpha} \in \widehat{\Delta}\right\}
$$

the closed boundary faces of $\langle V\rangle_{\Delta}$ are

$$
\partial^{I}\langle V\rangle_{\Delta} \equiv\langle V\rangle_{\Delta} \cap V^{I}=\left\{v \in V^{I} \mid \beta_{\alpha}^{I}(v) \leq 0 \text { for all } \beta_{\alpha}^{I} \in \widehat{\Delta}^{I}\right\}
$$

for $I \subseteq \Delta$. Consider the set obtained by flowing out orthogonally from $\partial^{I}\langle V\rangle_{\Delta}$ via the cone $V_{I}(0)$ :

$$
\langle V\rangle_{I} \equiv V_{I}(0)+\partial^{I}\langle V\rangle_{\Delta}
$$

In other words,

$$
\langle V\rangle_{I}=\left\{v \in V \mid \gamma_{I}(v)>0 \text { for all } \gamma_{I} \in \Delta_{I} \text {, and } \beta_{\alpha}^{I}(v) \leq 0 \text { for all } \beta_{\alpha} \in \widehat{\Delta}^{I}\right\}
$$

At one extreme, $\langle V\rangle_{\emptyset}$ is the open strictly dominant cone, while at the other, $\langle V\rangle_{\Delta}$ is the negative of the closed codominant cone. In general, by one of Langlands's "geometric lemmas", the various $\langle V\rangle_{I}$ 's are disjoint and fill up $V$ (see Figure 3 for an example):

Lemma 3.4 ([32, Lemma 4.4], [13, IV, §6.11)]. $V=\coprod_{I \subseteq \Delta}\langle V\rangle_{I}$. 


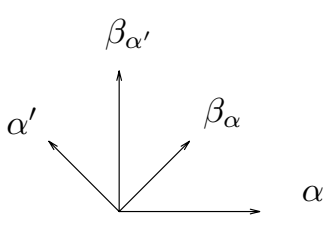

(a) $\Delta$ and $\widehat{\Delta}$ for $B_{2}$

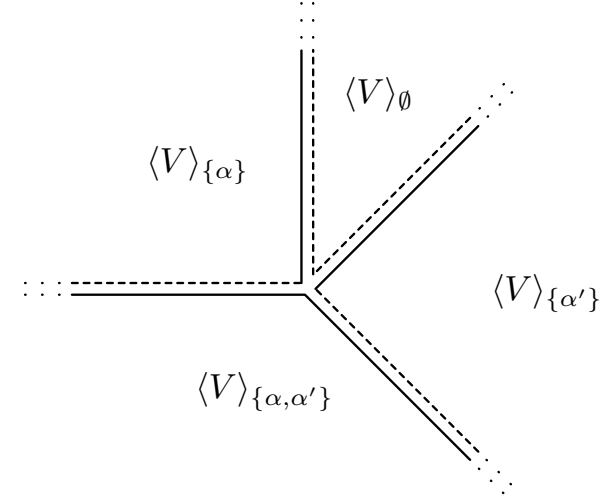

(b) $V=\coprod_{I \subseteq \Delta}\langle V\rangle_{I}$

(The tiles are separated slightly to indicate the boundaries.)

Figure 3.

The tiling for the root system $B_{2}$

It is clear that this is a tiling of $V$ in the sense of Definition 2.1 (appropriately modified).

Lemma 3.5. For $K \subseteq \Delta$ the tiling for $\left(V_{K}, \Delta_{K}\right)$ is given by $\left\langle V_{K}\right\rangle_{I_{K}}=\langle V\rangle_{I} \cap V_{K}$ for $I_{K} \subseteq \Delta_{K}$. Here $I \supseteq K$ and $I_{K}$ is its projection (excluding 0$)$ into $\Delta_{K}$.

For $K \subseteq \Delta$ the tiling for $\left(V^{K}, \Delta^{K}\right)$ is given by $\left\langle V^{K}\right\rangle_{I}=\langle V\rangle_{I}^{K}$ for $I \subseteq \Delta^{K}$. Here $\langle V\rangle_{I}^{K}$ is the image of $\langle V\rangle_{I}$ under the orthogonal projection onto $V^{K}$.

Proof. For $I \supseteq K$, the decomposition (3.2) of $V_{K}$ associated to $I_{K} \subseteq \Delta_{K}$ is $V_{K}=$ $V_{I} \oplus\left(V^{I} \cap V_{K}\right)$. The lemma for $V_{K}$ follows by intersecting this with (3.3). Now the decomposition of $V^{K}$ associated to $I \subseteq \Delta^{K}$ is $V^{K}=\left(V_{I} \cap V^{K}\right) \oplus V^{I}$. Again the lemma follows from this and (3.3); it is only necessary to check that $v \mapsto v^{K}$ takes $V_{I}(0)$ onto $\left(V_{I} \cap V^{K}\right)(0)=\left\{v^{K} \in V_{I} \cap V^{K} \mid \gamma_{I}\left(v^{K}\right)>0\right.$ for all $\left.\gamma_{I} \in \Delta_{I}^{K}\right\}$. To check this, observe that $\gamma_{I}\left(v^{K}\right)=\gamma_{I}(v)$ for $\gamma_{I} \in \Delta_{I}^{K}$, so the image of $V_{I}(0)$ lies in $\left(V_{I} \cap V^{K}\right)(0)$. Conversely, to show any $v^{K} \in\left(V_{I} \cap V^{K}\right)(0)$ is in the image, we need to find $v=v_{K}+v^{K} \in V_{I}(0)$; in other words, we need $\gamma_{I}\left(v_{K}\right)+\gamma_{I}\left(v^{K}\right)>0$ for all $\gamma_{I} \in \Delta_{I} \backslash \Delta_{I}^{K}$. Such a $v_{K} \in V_{K}$ can be found since $\Delta_{I} \backslash \Delta_{I}^{K}$ restricts to a basis of $V_{K}^{*}$.

We now extend this tiling of $V$ to one of a bordification $\bar{V}$. There is an isomorphism $V \cong \mathbb{R}^{\Delta}$ via the linear coordinates $v \mapsto(\alpha(v))_{\alpha \in \Delta}$. Define $\bar{V} \cong(\mathbb{R} \cup\{\infty\})^{\Delta}$ to be the semigroup obtained by allowing these coordinates independently to attain $\infty$. $\bar{V}$ is given an analytic structure by means of the coordinates $v \mapsto\left(e^{-\alpha(v)}\right)_{\alpha \in \Delta}$. (With this definition, the diffeomorphism exp $: \mathfrak{a}_{P} \rightarrow A_{P}$ extends to an analytic diffeomorphism exp : $\overline{\mathfrak{a}}_{P} \rightarrow \bar{A}_{P}$.) For $I \subseteq \Delta$, define $\bar{V}_{I}$ similarly with respect to the basis $\Delta_{I}$ of $V_{I}^{*}$. Clearly there is an analytic embedding of semigroups $\bar{V}_{I} \subseteq \bar{V}$, 


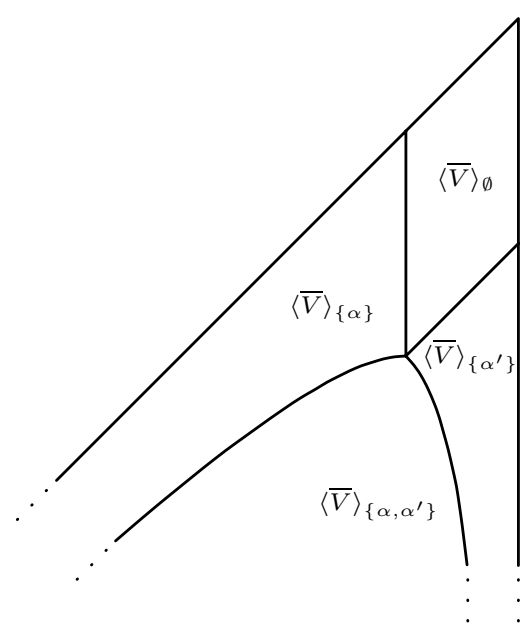

Figure 4.

$\bar{V}=\coprod_{I \subseteq \Delta}\langle\bar{V}\rangle_{I}$ for the root system $B_{2}$ (in analytic coordinates adjusted to be conformal at the origin)

although the product decomposition (3.2) does not extend to $\bar{V}$. It does however extend to a certain subset $V(I)$ :

Lemma 3.6. There is an analytic decomposition

$$
V(I) \equiv\{v \in \bar{V} \mid \alpha(v)<\infty \text { for all } \alpha \in I\} \cong \bar{V}_{I} \oplus V^{I} .
$$

Proof. The main issue is to show that the projection $v \mapsto v_{I}$ extends analytically to the set in question; in other words, we need to show that $v \mapsto e^{-\gamma_{I}(v)}$ for $\gamma_{I} \in \Delta_{I}$ is analytic on this set. Now note that for any codominant $\lambda=\sum c_{\alpha} \alpha \in V^{*}$, there is a continuous map $e^{-\lambda}: \bar{V} \rightarrow \mathbb{R}$. The function $e^{-\lambda}$ is analytic at $v$ if $\alpha(v)<\infty$ for all $\alpha$ with $c_{\alpha}>0$ and nonintegral. Thus by Remark 3.3(i), $e^{-\gamma_{I}}=e^{-\gamma} e^{\gamma^{I}}$ for $\gamma \notin I$ is analytic where needed.

We can thus define $\langle\bar{V}\rangle_{I} \subseteq V(I)$ by

$$
\begin{aligned}
\langle\bar{V}\rangle_{I} & \equiv \bar{V}_{I}(0)+\partial^{I}\langle V\rangle_{\Delta} \\
& =\left\{v \in \bar{V} \mid \gamma_{I}(v)>0 \text { for all } \gamma_{I} \in \Delta_{I}, \text { and } \beta_{\alpha}^{I}(v) \leq 0 \text { for all } \beta_{\alpha}^{I} \in \widehat{\Delta}^{I}\right\}
\end{aligned}
$$

similarly to (3.3) and (3.4); see Figure 4 for an example drawn using analytic coordinates.

Proposition 3.7. $\bar{V}=\coprod_{I \subseteq \Delta}\langle\bar{V}\rangle_{I}$ is an analytic tiling. 
Proof. For $K \subseteq \Delta$, let $e(K)=\{v \in \bar{V} \mid \alpha(v)=\infty$ for $\alpha \notin K$, and $\alpha(v)<$ $\infty$ for $\alpha \in K\}$. To show that the sets $\langle\bar{V}\rangle_{I}$ are disjoint and exhaust $\bar{V}$ it suffices to show that $e(K)=\coprod_{I \subseteq \Delta}\langle\bar{V}\rangle_{I} \cap e(K)$ for all $K \subseteq \Delta$. But $e(K) \stackrel{\sim}{\rightarrow} V^{K}$ under the projection $v \mapsto v^{K}$, while $\langle\bar{V}\rangle_{I} \cap e(K) \subseteq V(I) \cap e(K)$ is empty unless $I \subseteq K$, in which case it projects to $\langle V\rangle_{I}^{K}$. Now apply Lemma 3.5. It is clear that the conditions of Definition 2.1 are satisfied and the fact that $\langle\bar{V}\rangle_{I}$ is an analytic submanifold follows from (3.5) and Lemma 3.6.

In the case $(V, \Delta)=\left(\mathfrak{a}_{P}, \Delta_{P}\right)$ we apply the analytic isomorphism exp : $\overline{\mathfrak{a}}_{P} \rightarrow$ $\bar{A}_{P}$ to obtain:

Corollary 3.8. $\bar{A}_{P}=\coprod_{R \supseteq P}\left\langle\bar{A}_{P}\right\rangle_{R}$ is an analytic tiling, where $\left\langle\bar{A}_{P}\right\rangle_{R} \equiv$ $\exp \left(\left\langle\mathfrak{a}_{P}\right\rangle_{\Delta_{P}^{R}}\right)$.

\section{Construction of tilings, II: Tilings associated to a parabolic $P$}

Let $\mathbf{b} \in \mathcal{B}$ be a parameter. In this second part of the construction we transfer the tiling of $\bar{A}_{P}$ to a tiling of $X(P)$ with parameter $\mathbf{b}$. This is done by shifting the tiling by $b_{P}$ and then using the decomposition $X(P) \cong \bar{A}_{P} \times e(P)$.

Thus for $R \in \mathcal{P}$ with $R \supseteq P$ we define

$$
X(P)_{R}=b_{P} \cdot\left\langle\bar{A}_{P}\right\rangle_{R} \times e(P) ;
$$

when $R=G$, we simply write $X(P)_{0}$ for $X(P)_{G}$. Let

$$
\partial^{R} X(P)_{0}=b_{P} \cdot \partial^{R}\left\langle A_{P}\right\rangle_{0} \times e(P) .
$$

It is useful to describe these sets directly by inequalities. The last part of equation (3.5) translates to

$$
\begin{aligned}
& X(P)_{R}=\left\{z \in X(P) \mid a_{P}(z)^{\gamma_{R}}>b_{P}^{\gamma_{R}} \text { for all } \gamma_{R} \in \Delta_{R}\right. \text { and } \\
& \left.\qquad a_{P}(z)^{\beta_{Q}^{R}} \leq b_{P}^{\beta_{Q}^{R}} \text { for all } \beta_{Q}^{R} \in \widehat{\Delta}_{P}^{R}\right\} .
\end{aligned}
$$

Since $a_{P}^{\beta_{Q}}=a_{Q}^{\beta_{Q}}$ for $P \subseteq Q$ and the same holds true for the parameters, we may rephrase this in terms of the distance functions $\left(a_{Q}^{\beta_{Q}}\right)_{Q \in \mathcal{P}_{1}}$ to the maximal boundary faces. In particular we have:

$$
\begin{gathered}
X(P)_{0}=\left\{z \in X \mid a_{Q}(z)^{\beta_{Q}} \leq b_{Q}^{\beta_{Q}} \text { for all } Q \in \mathcal{P}_{1} \text { with } Q \supseteq P\right\}, \\
\partial^{R} X(P)_{0}=\left\{z \in X(P)_{0} \mid a_{Q}(z)^{\beta_{Q}}=b_{Q}^{\beta_{Q}} \text { for all } Q \in \mathcal{P}_{1} \text { with } Q \supseteq R\right\} .
\end{gathered}
$$


Proposition 4.1. $X(P)=\coprod_{R \supseteq P} X(P)_{R}$ is an analytic tiling of $X(P)$ depending analytically on the parameter $\mathbf{b}$.

Definition. $\left\{X(P)_{R}\right\}_{R \supseteq P}$ is called the tiling associated to $P$.

Proof. This follows from Corollary 3.8. For Definition 2.1(ii), one notes that

$$
\partial^{R} X(P)_{0}=\partial^{R} X(R)_{0} \cap X(P)_{0} \subseteq \partial^{R} X(R)_{0}=\left\{b_{R}\right\} \times e(R)
$$

(use (4.2) and (4.3) for the first equality). For Definition 2.1(iii) one must recall that the left action of $\bar{A}_{R}$ on $\bar{A}_{P}$ corresponds under $X(P) \cong \bar{A}_{P} \times e(P)$ with the geodesic action of $\bar{A}_{R}$ on $X(P)$.

\section{Construction of tilings, III: Tilings of $\bar{X}$ by refinement}

In this final part of the construction we show that for sufficiently large $\Gamma$-invariant parameters $\mathbf{b}$ the intersection of the tilings associated to all parabolics $P \in \mathcal{P}$ yields a tiling of $\bar{X}$. Thus for this section (and only for this section) we set

$$
\bar{X}_{R}=\bigcap_{P \subseteq R} X(P)_{R}
$$

and

$$
\partial^{R} X_{0}=\bigcap_{P \subseteq R} \partial^{R} X(P)_{0}
$$

(as usual we write $X_{0}$ for $\bar{X}_{G}$ ). In particular, $X_{0}$ is defined by the inequalities $a_{Q}(z)^{\beta_{Q}} \leq b_{Q}^{\beta_{Q}}$ for all maximal parabolics $Q$. The plan is to use an estimate from reduction theory to show that within each element of certain open covers, (5.1) agrees with the tiling associated with some $P$ provided $\mathbf{b}$ is large.

Definition 5.1 (compare with $[10, \S 5.3]^{3}$ ). A cylindrical set (relative to a parabolic $P$ ) is a set of the form

$$
W_{P} \equiv A_{P}\left(s_{P}\right) \times O_{P}
$$

where $s_{P} \in A_{P}$ and $O_{P} \subseteq e(P)$ is an open, $\Gamma_{P}$-invariant and $N_{P}$-invariant subset such that $\Gamma_{P} \backslash O_{P}$ is relatively compact. Similarly the subset $\bar{W}_{P} \subseteq \bar{X}$ defined

\footnotetext{
${ }^{3}$ In [10] $O_{P}$ is itself relatively compact and not $\Gamma_{P}$-invariant. A cylindrical set here is thus the union of the $\Gamma_{P}$-translates of a cylindrical set in [10]. This is more convenient for our purposes; note however that our definition depends on the choice of arithmetic subgroup $\Gamma$.
} 
using $\bar{A}_{P}$ will be called cylindrical. An open cover $\mathcal{W}=\left\{W_{P}\right\}_{P \in \mathcal{P}}$ of $X$ consisting of cylindrical sets is called a cylindrical cover. The cover is said to be $\Gamma$-invariant if $\gamma \cdot W_{P}=W_{\gamma_{P}}$ for $\gamma \in \Gamma$.

Remark. It follows from reduction theory $[9, \S 13.1]$ that $\Gamma$-invariant cylindrical covers exist. For example, one may take $W_{P}$ to be empty except for $P$ minimal, where it would be $\Gamma_{P}$ times a large Siegel set.

The estimate is simplest to state using the normalized functions

$$
\hat{a}_{P}(z) \equiv a_{P}(z) / b_{P}
$$

Proposition 5.2. Let $\mathcal{W}=\left\{W_{P}\right\}_{P \in \mathcal{P}}$ be a $\Gamma$-invariant cylindrical cover. If $\mathbf{b} \in \mathcal{B}^{\Gamma}$ is sufficiently large, then for all $P \in \mathcal{P}$ and for all $Q \in \mathcal{P}_{1}$ with $Q \nsupseteq P$,

$$
\hat{a}_{Q}(z)^{\beta_{Q}}<\hat{a}_{P}(z)^{\lambda_{Q, P}} \quad\left(z \in W_{P}\right),
$$

where $\lambda_{Q, P} \in \mathfrak{a}_{P}^{*}$ is dominant (depending only on the type of $Q$ and $P$ ) and $\beta_{Q}-\lambda_{Q, P}$ is nonzero and codominant.

Proof. Let $\alpha$ be the unique element of $\Delta \backslash \Delta^{Q}$ and define $\lambda_{Q, P} \equiv\left(\beta_{Q}-\epsilon \alpha\right)_{P}$, where $\epsilon$ will be determined. For $\epsilon>0$ small, the functional $\beta_{Q}-\epsilon \alpha$ is dominant, and thus by Lemma 3.2 so is $\lambda_{Q, P}$. In addition,

$$
\beta_{Q}-\lambda_{P, Q}= \begin{cases}\beta_{Q}^{P} & \text { if } \alpha \in \Delta^{P}, \\ \epsilon \alpha_{P} & \text { if } \alpha \notin \Delta^{P},\end{cases}
$$

which is nonzero and by Lemma 3.2 is codominant.

To prove the estimate, note that by the proof of Proposition 2.6 the normalized functions satisfy the transformation law

$$
\hat{a}_{g P}(g z)=\left(c(g, P) b_{P} / b_{g_{P}}\right) \hat{a}_{P}(z)
$$

for $g \in G_{\mathbb{Q}}$; in particular, by the $\Gamma$-invariance of $\mathbf{b}$ (see (2.4)),

$$
\hat{a}_{\gamma P}(\gamma z)=\hat{a}_{P}(z) \quad(\gamma \in \Gamma) .
$$

Thus it suffices to fix $P$ belonging to a finite set of representatives of $\Gamma$-conjugacy classes; choose a minimal parabolic for which $P$ is standard. The maximal parabolics $Q \nsupseteq P$ may be enumerated as $Q_{0}^{g \gamma}$, where $Q_{0}$ is standard, $g \in G_{\mathbb{Q}}$ ranges over a finite set, and $\gamma \in \Gamma$ is restricted such that $g \gamma \notin Q_{0}$ in the case that $Q_{0} \supseteq P$. Then $\hat{a}_{Q}(z)^{\beta_{Q}}=C \cdot \hat{a}_{Q_{0}}(g \gamma z)^{\beta_{Q}}$ by (5.3) and (5.4), where $C>0$ depends only 
on $g, Q_{0}$, and $\mathbf{b}$. A result of reduction theory [43, Lemma 4.9$]^{4}$ shows that this is bounded by $C \cdot \hat{a}_{P}(z)^{\beta_{Q}-m \alpha}$ with $m>0$ (if $Q_{0} \supseteq P$, use the last equation of the proof). But since $\hat{a}_{P} \in A_{P}\left(s_{P} / b_{P}\right)$, we have $\hat{a}_{P}^{\beta_{Q}-m \alpha}=\hat{a}_{P}^{(\epsilon-m) \alpha_{P}} \hat{a}_{P}^{\lambda_{Q}, P} \leq C \cdot \hat{a}_{P}^{\lambda_{Q, P}}$ provided we choose $\epsilon<m$.

To see that the constant $C$ may be chosen less than one, note that if $\mathbf{b}$ is replaced by $t \cdot \mathbf{b}$, then $C$ scales as $t^{-\left(\beta_{Q}-\lambda_{Q, P}\right)}$. This can be made arbitrarily small provided $t$ is sufficiently dominant.

Corollary 5.3. Let $\mathcal{W}=\left\{W_{P}\right\}_{P \in \mathcal{P}}$ be a $\Gamma$-invariant cylindrical cover. If $\mathbf{b} \in \mathcal{B}^{\Gamma}$ is sufficiently large, then for all $P, P^{\prime} \in \mathcal{P}$ and for all $Q \in \mathcal{P}_{1}$ with $Q \nsupseteq P$,

$$
\hat{a}_{Q}(z)^{\beta_{Q}}<\hat{a}_{P^{\prime}}(z)^{\lambda_{Q, P, P^{\prime}}} \quad\left(z \in W_{P} \cap W_{P^{\prime}}\right),
$$

where $\lambda_{Q, P, P^{\prime}} \in \mathfrak{a}_{P^{\prime}}^{*}$ is dominant and $\beta_{Q}-\lambda_{Q, P, P^{\prime}}$ is nonzero and codominant.

Proof. Write $\lambda_{Q, P}=\sum_{P \subseteq Q^{\prime} \in \mathcal{P}_{1}} c_{Q^{\prime}} \beta_{Q^{\prime}}$, where $c_{Q^{\prime}} \geq 0$. Then Proposition 5.2 yields the estimate

$$
\hat{a}_{Q}(z)^{\beta_{Q}}<\prod_{Q^{\prime} \supseteq P} \hat{a}_{Q^{\prime}}(z)^{c_{Q^{\prime}} \beta_{Q^{\prime}}} \quad\left(z \in W_{P}\right) .
$$

Now apply Proposition 5.2 again to estimate in $W_{P^{\prime}}$ those factors on the right-hand side for which $Q^{\prime} \nsupseteq P^{\prime}$. This yields (5.5), where

$$
\lambda_{Q, P, P^{\prime}}=\sum_{\substack{Q^{\prime} \supseteq P \\ Q^{\prime} \supseteq P^{\prime}}} c_{Q^{\prime}} \beta_{Q^{\prime}}+\sum_{\substack{Q^{\prime} \supseteq P \\ Q^{\prime} \nsupseteq P^{\prime}}} c_{Q^{\prime}} \lambda_{Q^{\prime}, P^{\prime}} .
$$

Now $\lambda_{Q, P, P^{\prime}} \in \mathfrak{a}_{P^{\prime}}^{*}$ is clearly dominant and furthermore

$$
\begin{aligned}
\beta_{Q}-\lambda_{Q, P, P^{\prime}} & =\left(\lambda_{Q, P}-\lambda_{Q, P, P^{\prime}}\right)+\left(\beta_{Q}-\lambda_{Q, P}\right) \\
& =\sum_{Q^{\prime} \supseteq P} c_{Q^{\prime}}\left(\beta_{Q^{\prime}}-\lambda_{Q^{\prime}, P^{\prime}}\right)+\left(\beta_{Q}-\lambda_{Q, P}\right)
\end{aligned}
$$

is nonzero and codominant. $Q^{\prime} \nsupseteq P^{\prime}$

We will also need one simple lemma which will be useful later. For a cylindrical set $\bar{W}_{P}=\bar{A}_{P}\left(s_{P}\right) \times O_{P}$, write $b_{P} \sim \bar{W}_{P}$ if $b_{P} \in \bar{A}_{P}\left(s_{P}\right)$.

Lemma 5.4. Let $\bar{W}_{P}$ be a cylindrical set such that $b_{P} \sim \bar{W}_{P}$. Then for $R \supseteq P$,

$$
\bar{A}_{R}(1) \mathbf{o}\left(\partial^{R} X(P)_{0} \cap \bar{W}_{P}\right)=X(P)_{R} \cap \bar{W}_{P}=X(P)_{R} \cap\left(\bar{A}_{R} \mathbf{o} \bar{W}_{P}\right) .
$$

\footnotetext{
4 The result in [43] was stated for $G$ the group of automorphisms of a self-adjoint homogeneous cone; aside from the notation, the result and proof apply to the general case. The proof uses results on functions of type $(P, \chi)$ from $[9, \S 14]$ and is similar to arguments in $[6, \S \S 7.5-7.8]$.
} 
Proof. It is clear from the definitions that each of these sets is contained in the next. So let $z \in X(P)_{R} \cap\left(\bar{A}_{R} \mathbf{o} \bar{W}_{P}\right)$ and write $z=a \mathbf{o} y$ with $a \in \bar{A}_{R}(1)$ and $y \in \partial^{R} X(P)_{0}$; we need to show $y \in \bar{W}_{P}$. Since $\bar{A}_{R} \subseteq \bar{A}_{P}$, we have $q_{P}(y)=q_{P}(z) \in$ $O_{P}$. If $\alpha \in \Delta_{P}^{R}$ (and therefore is trivial on $\bar{A}_{R}$ ), then $a_{P}(y)^{\alpha}=a_{P}(z)^{\alpha} \geq s_{P}^{\alpha}$. If $\gamma \in \Delta_{P} \backslash \Delta_{P}^{R}$, then $\gamma^{R}$ is antidominant with respect to $\Delta_{P}^{R}$ (by Remark 3.3(i)) and so (since $\left.y \in \partial^{R} X(P)_{0}\right) a_{P}(y)^{\gamma^{R}} \geq b_{P}^{\gamma^{R}}$. On the other hand, $a_{P}(y)^{\gamma_{R}}=b_{P}^{\gamma_{R}}$. Thus $a_{P}(y)^{\gamma} \geq b_{P}^{\gamma} \geq s_{P}^{\gamma}$ since $b_{P} \sim \bar{W}_{P}$.

We can now show that (5.1) agrees in $\bar{W}_{P}$ with the tiling associated to $P$ provided $\mathbf{b}$ is large.

Proposition 5.5. Let $\mathcal{W}=\left\{\bar{W}_{P}\right\}_{P \in \mathcal{P}}$ be a $\Gamma$-invariant cylindrical cover of $\bar{X}$. If $\mathbf{b} \in \mathcal{B}^{\Gamma}$ is sufficiently large, then for all $P, R \in \mathcal{P}$,

(i) $b_{P} \sim \bar{W}_{P}$,

(ii) $\bar{X}_{R} \cap \bar{W}_{P}= \begin{cases}X(P)_{R} \cap \bar{W}_{P} & \text { for } P \subseteq R, \\ \emptyset & \text { for } P \nsubseteq R,\end{cases}$

(iii) $\partial^{R} X_{0} \cap \bar{W}_{P}= \begin{cases}\partial^{R} X(P)_{0} \cap \bar{W}_{P} & \text { for } P \subseteq R, \\ \emptyset & \text { for } P \nsubseteq R,\end{cases}$

(iv) $\bar{X}_{R}=\bar{A}_{R}(1) \mathbf{o} \partial^{R} X_{0}$.

Remark. In the case that $X$ is the semisimple part of a self-adjoint homogeneous cone, the case $R=G$ of (ii) is essentially [43, Proposition 4.4].

Proof. Clearly assertion (i) can be arranged making $\mathbf{b}$ larger if necessary. Case $R=G$ of (ii) is equivalent (by (4.2)) to the inequalities $\hat{a}_{Q}(z)^{\beta_{Q}} \leq 1$ for $Q \supseteq P$ implying in $W_{P}$ the inequalities $\hat{a}_{Q}(z)^{\beta_{Q}} \leq 1$ for all $Q \nsupseteq P$. This implication follows by Proposition 5.2. In view of (4.3), we see that Proposition 5.2 implies (iii) by the same argument. (In the case $P \nsubseteq R$, use the fact that there exists a $Q \in \mathcal{P}_{1}$ with $Q \supseteq R$ and $Q \nsupseteq P$, together with the strict inequality.) Furthermore, the intersection of $X(P)_{R}=\bar{A}_{R}(1)$ o $\partial^{R} X(P)_{0}$ over all $P \subseteq R$ yields (iv).

We now consider (ii) where $R \neq G$. If $P \subseteq R$, (iii) and (iv) together with Lemma 5.4 show that $X(P)_{R} \cap \bar{W}_{P} \subseteq \bar{X}_{R} \cap \bar{W}_{P}$, and hence we have equality. If on the other hand $P \nsubseteq R$, first note that the case $P^{\prime} \subseteq R$ already treated together with Lemma 5.4 imply $\bar{X}_{R} \cap \bar{W}_{P^{\prime}}=X\left(P^{\prime}\right)_{R} \cap\left(\bar{A}_{R} \mathbf{o} \bar{W}_{P^{\prime}}\right) \supseteq \bar{X}_{R} \cap\left(\bar{A}_{R} \mathbf{o} \bar{W}_{P^{\prime}}\right)$. But $\left\{\bar{W}_{P^{\prime}}\right\}_{P^{\prime} \subseteq R}$ covers $e(R)$, and thus $\left\{\bar{A}_{R} \mathbf{o} \bar{W}_{P^{\prime}}\right\}_{P^{\prime} \subseteq R}$ covers $\bar{X}_{R}$. Thus it follows that $\bar{X}_{R} \subseteq \bigcup_{P^{\prime} \subset R} \bar{W}_{P^{\prime}}$ and so it suffices to show that $\bar{X}_{R} \cap \bar{W}_{P} \cap \bar{W}_{P^{\prime}}=\emptyset$ for all $P^{\prime} \subseteq R$. The application of Corollary 5.3 to $Q \in \mathcal{P}_{1}$ with $Q \supseteq R$ and $Q \nsupseteq P$ yields that $\hat{a}_{P^{\prime}}^{\beta_{Q}-\lambda_{Q, P, P^{\prime}}}<1$ on $\bar{W}_{P} \cap \bar{W}_{P^{\prime}}$. We claim though that $\hat{a}_{P^{\prime}}^{\beta_{Q}-\lambda_{Q, P, P^{\prime}}}>1$ on $\bar{X}_{R} \cap \bar{W}_{P^{\prime}}=X\left(P^{\prime}\right)_{R} \cap \bar{W}_{P^{\prime}}$, which will finish the proof. To see the claim, note that Lemma 3.2 implies that $\left(\beta_{Q}-\lambda_{Q, P, P^{\prime}}\right)^{R}=-\lambda_{Q, P, P^{\prime}}^{R}$ is antidominant with respect to $\Delta_{P^{\prime}}^{R}$, and that $\left(\beta_{Q}-\lambda_{Q, P, P^{\prime}}\right)_{R}$ is (nonzero) codominant. The claim 
then follows from (4.1).

Let us formalize this interrelationship between a tiling and a cylindrical cover.

Definition 5.6. If $\mathcal{W}=\left\{\bar{W}_{P}\right\}_{P \in \mathcal{P}}$ is a cylindrical cover, a tiling $\left\{\bar{X}_{R}\right\}_{R \in \mathcal{P}}$ with parameter $\mathbf{b}$ is said to be $\mathcal{W}$-adapted (or adapted to $\mathcal{W}$ ) if for all $P, R \in \mathcal{P}$,

(i) $b_{P} \sim \bar{W}_{P}$, and

(ii) $\bar{X}_{R} \cap \bar{W}_{P}= \begin{cases}X(P)_{R} \cap \bar{W}_{P} & \text { for } P \subseteq R, \\ \emptyset & \text { for } P \nsubseteq R .\end{cases}$

With this terminology we summarize what we have done in the

Theorem 5.7. Let $\mathcal{W}=\left\{\bar{W}_{P}\right\}_{P \in \mathcal{P}}$ be a $\Gamma$-invariant cylindrical cover of $\bar{X}$. If $\mathbf{b} \in \mathcal{B}^{\Gamma}$ is sufficiently large, there exists a unique analytic family of $\mathcal{W}$-adapted analytic tilings of $\bar{X}$ with parameters $t \cdot \mathbf{b}, t \in \operatorname{cl}(A(1))$.

Proof. Apply Propositions 4.1 and 5.5; uniqueness follows from Corollary 2.5.

We have stated our final result this way since, in working with these tilings, it will be easier to use $\mathcal{W}$-adaptedness rather than the original construction (5.1). In fact, all $\Gamma$-invariant tilings in the sense of Definition 2.1 (not necessarily constructed as in (5.1)) are adapted to some $\Gamma$-invariant cylindrical cover $\mathcal{W}$. This will be proved in the beginning of $\S 9$, where we also study the space of parameters b for which a tiling exists. Thus the following sections actually apply to all tilings. Of course, if one simply wishes to work with the tilings we have constructed above (for $\mathbf{b}$ large), this remark and $\S 9$ may be safely ignored.

There remains however the interesting

Question 5.8. Can every tiling (with parameter $\mathbf{b}$ not necessarily large) be expressed as in (5.1)? More particularly, is the central tile of any tiling defined by the inequalities $a_{Q}(z)^{\beta_{Q}} \leq b_{Q}^{\beta_{Q}}$ for all maximal parabolics $Q$ ?

\section{Retractions and diffeomorphisms}

We now consider retractions onto central tiles. Given a tiling of $\bar{X}$, the desired retraction projects $\bar{X}_{P}=\bar{A}_{P}(1)$ o $\partial^{P} X_{0}$ back along the $\bar{A}_{P}(1)$-orbits onto $\partial^{P} X_{0}$ (see Figure 5). By varying the parameter, one may obtain a family of retractions converging to the identity. We also construct a diffeomorphism $\bar{X} \rightarrow X_{0}$ for use in a later paper (see Figure 6).

Theorem 6.1. For $\mathbf{b} \in \mathcal{B}^{\Gamma}$ fixed and for all $t \in \operatorname{cl}(A(1))$, let $\left\{\bar{X}_{P, t}\right\}_{P \in \mathcal{P}}$ be a 


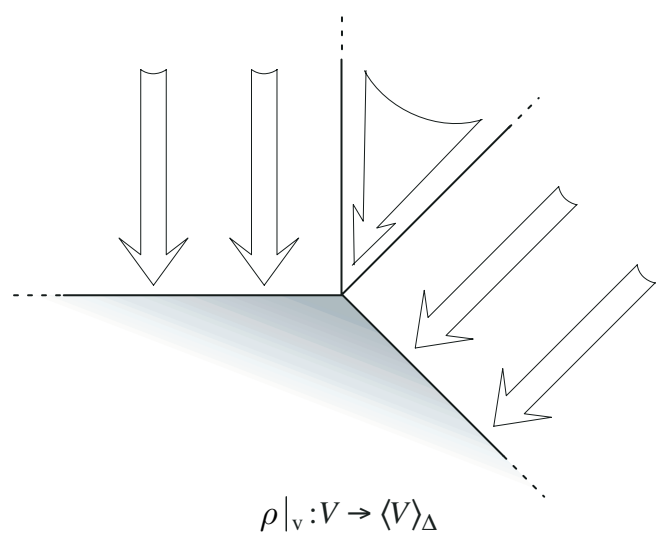

Figure 5.

The retraction of $V$ (the image is shaded)

tiling of $\bar{X}$ with parameter $t \cdot \mathbf{b}$.

(1) For all $t \in \operatorname{cl}(A(1))$, there exists a unique $\Gamma$-equivariant piecewise-analytic retraction $r_{t}: \bar{X} \rightarrow X_{0, t}$ satisfying $r_{t}\left(\bar{A}_{P}(1) \mathbf{o} y\right)=y$ for $y \in \partial^{P} X_{0, t}$ and $P \in \mathcal{P}$.

(2) For all $t \in A(1)$, there exists a unique $\Gamma$-equivariant piecewise-analytic diffeomorphism $s_{t}: \bar{X} \rightarrow X_{0, t}$ such that for all $P \in \mathcal{P}$ :

(i) $s_{t}$ preserves the $\bar{A}_{P}(1)$-orbits in $\bar{X}_{P, 1}$.

(ii) The family of diffeomorphisms induced on the $\bar{A}_{P}(1)$-orbits in $\bar{X}_{P, 1}$ is constant with respect to the canonical cross-sections.

(iii) In terms of the coordinates $a \mapsto\left(a^{-\alpha}\right)_{\alpha \in \Delta_{P}}$, each coordinate function of the diffeomorphism induced on $\bar{A}_{P}(1)$ is the exponential of a polynomial having degree at most 1 in each variable.

Both $r_{t}$ and $s_{t}$ depend piecewise-analytically on $t$. As $t$ tends to infinity under the action of a strictly dominant 1-parameter subgroup, $r_{t}$ and $s_{t}$ converge to the identity; as $t$ tends to $1, s_{t}$ converges to $r_{1}$.

The construction can be broken into the same three steps as our construction of tilings. First we define models on $(\bar{V}, \Delta)=\left(\overline{\mathfrak{a}}_{P}, \Delta_{P}\right)$ for the retraction and diffeomorphism. Let $\bar{V}=\coprod_{I \subseteq \Delta}\langle\bar{V}\rangle_{I}$ be the tiling constructed in $\S 3$. The model for the desired retraction is the piecewise-analytic map $\rho: \bar{V} \rightarrow\langle V\rangle_{\Delta}$ defined by

$$
\rho(v)=v^{I} \in \partial^{I}\langle V\rangle_{\Delta} \quad \text { if } v \in\langle\bar{V}\rangle_{I}
$$

In $\langle\bar{V}\rangle_{I}=\bar{V}_{I}(0)+\partial^{I}\langle V\rangle_{\Delta}$ this is the projection map onto the second factor; see Figure 5 . 

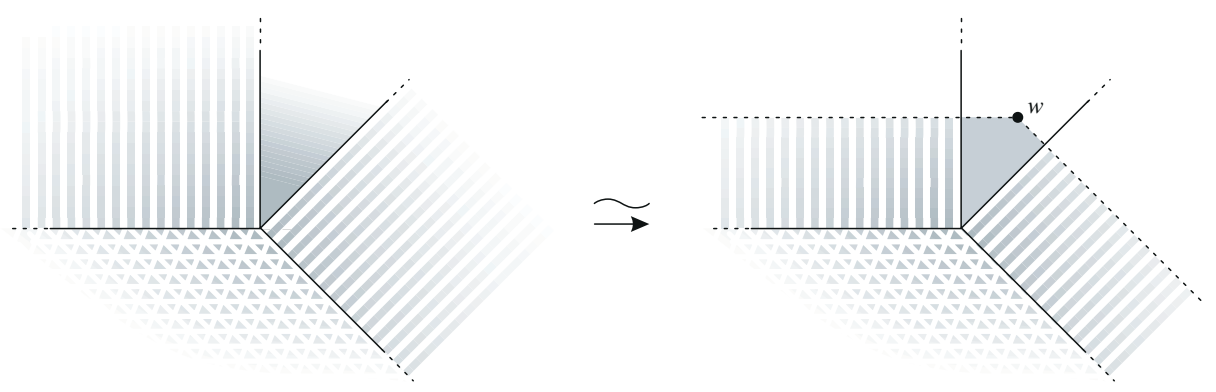

Figure 6 .

The diffeomorphism $\left.\sigma_{w}\right|_{V}: V \stackrel{\sim}{\longrightarrow}+\operatorname{Int}\left(\langle V\rangle_{\Delta}\right)$. (The shading design in each tile $\langle V\rangle_{I}$ suggests the corresponding fibers of $\rho$.)

For the model of the diffeomorphism, a more complicated construction is required:

Lemma 6.2. Let $w \in V(0)$. There exists a unique piecewise-analytic diffeomorphism $\sigma_{w}: \bar{V} \stackrel{\sim}{\rightarrow} w+\langle V\rangle_{\Delta}$ with the following properties:

(i) For $v=v_{I}+v^{I} \in\langle\bar{V}\rangle_{I}, \sigma_{w}(v)=\sigma_{w}\left(v_{I}\right)+v^{I}$.

(ii) In $\operatorname{cl}\left(\langle\bar{V}\rangle_{\emptyset}\right)=\operatorname{cl}(\bar{V}(0)), \sigma_{w}$ is given by a polynomial in the variables $x_{\alpha}=$ $e^{-\alpha(v)}(\alpha \in \Delta)$ with degree at most 1 in each variable.

For $K \subseteq \Delta$, the restriction of $\sigma_{w}$ to $\bar{V}_{K}$ is the corresponding diffeomorphism associated to $\left(\bar{V}_{K}, \Delta_{K}\right)$ and $w_{K}$.

Proof. By (i), it suffices to define an analytic diffeomorphism $\sigma_{w}$ on $\mathrm{cl}(\bar{V}(0))$ such that $\sigma_{w}$ preserves the closed boundary faces $\operatorname{cl}\left(\bar{V}_{I}(0)\right), I \subset \Delta$. The required polynomial is given by

$$
v \mapsto \sum_{I \subseteq \Delta}\left(\prod_{\alpha \in \Delta \backslash I}\left(1-x_{\alpha}\right) \prod_{\alpha \in I} x_{\alpha}\right) w_{I} .
$$

The uniqueness of the coefficient of $\prod_{\alpha \in K} x_{\alpha}$ follows by induction on $|K|$ and $|\Delta|$. The final assertion of the lemma follows from uniqueness and Lemma 3.5.

Remark. The reason a polynomial of total degree greater than 1 is required in (6.2) is that whereas $\mathrm{cl}(\bar{V}(0))$ is a "parallelpiped" relative to the analytic coordinates $\left(x_{\alpha}\right)$ (see Figure 4), the image $\left(w+\langle V\rangle_{\Delta}\right) \cap \operatorname{cl}(\bar{V}(0))$ relative to linear coordinates is not in general (see Figure 6).

We denote the induced maps on $\bar{A}_{P}$ also by $\rho$ and $\sigma_{t}$, where $w=\log t_{P}$. 
The second step is to shift and transfer these models to $X(P) \cong \bar{A}_{P} \times e(P)$. Let $\left\{X(P)_{R, t}\right\}_{R \supseteq P}$ be the tiling associated to $P$ with parameter $t \cdot \mathbf{b}$. Define $r_{t}^{(P)}: X(P) \rightarrow X(P)_{0, t}$ by

$$
r_{t}^{(P)}(a, y)=\left(t_{P} b_{P} \rho\left(\left(t_{P} b_{P}\right)^{-1} a\right), y\right),
$$

and $s_{t}^{(P)}: X(P) \rightarrow X(P)_{0, t}$ by

$$
s_{t}^{(P)}(a, y)=\left(b_{P} \sigma_{t}\left(b_{P}^{-1} a\right), y\right) .
$$

Finally in the third step we define $r_{t}: \bar{X} \rightarrow X_{0, t}$ and $s_{t}: \bar{X} \rightarrow X_{0, t}$ by

$$
\begin{aligned}
& r_{t}(z)=r_{t}^{(R)}(z) \quad\left(z \in \bar{X}_{R, t}\right), \\
& s_{t}(z)=s_{t}^{(R)}(z) \quad\left(z \in \bar{X}_{R, 1}\right) \text {. }
\end{aligned}
$$

Proof of Theorem 6.1. Let $\mathcal{W}=\left\{\bar{W}_{P}\right\}_{P \in \mathcal{P}}$ be a $\Gamma$-invariant cylindrical cover to which our tilings are adapted for all $t$. (For the family of tilings constructed in Theorem 5.7, such a cover was part of the construction; in general we will prove such a cover exists later in Theorem 9.6.) For any $P \in \mathcal{P}$ we claim that $r_{t}=r_{t}^{(P)}$ and $s_{t}=s_{t}^{(P)}$ in the open set $\bar{W}_{P}$. To see this, first note that by adaptedness, any $z \in \bar{W}_{P}$ must belong to $X(P)_{R, t}$ for some $R \supseteq P$. Then we compute $r_{t}(z)=r_{t}^{(R)}(z)=\left(t_{R} b_{R}, q_{R}(z)\right.$ ) (under $X \cong A_{R} \times e(R)$ ), whereas $r_{t}^{(P)}(z)=\left(t_{P} b_{P}\left(\left(t_{P} b_{P}\right)^{-1} a_{P}(z)\right)^{R}, q_{P}(z)\right)=\left(t_{R} b_{R} a_{P}(z)^{R}, q_{P}(z)\right)$ (under $\left.X \cong A_{P} \times e(P)\right)$. These two expressions are equal since ${ }^{0} R x=A_{P}^{R} \mathbf{o}^{0} P x$. The claim for the diffeomorphism follows similarly by using the last assertion of Lemma 6.2.

By the claim we are reduced to proving the theorem for the maps $r_{t}^{(P)}$ and $s_{t}^{(P)}$. These maps are clearly piecewise-analytic and have analytic dependence on $t$. It is also easy to see they have the determining properties; for $s_{t}^{(P)}$ use Lemma 6.2.

Now assume that $t$ is tending to infinity under the action of a strictly dominant subgroup and consider $z \in e(R) \subset X(P)$ for some $R \supseteq P$. By Lemma 3.2, $t_{P}^{R}$ is tending to infinity under the action of a strictly dominant (and hence strictly codominant) 1-parameter subgroup in $A_{P}^{R}$, so eventually $a_{P}(z)^{\beta_{Q}^{R}} \leq\left(t_{P} b_{P}\right)^{\beta_{Q}^{R}}$ for all $\beta_{Q}^{R} \in \widehat{\Delta}_{P}^{R}$. Thus by (4.1), $z \in X(P)_{R, t} \cap e(R)$ for $t$ sufficiently large, in which case $r_{t}^{(P)}(z)=\left(t_{R} b_{R} a_{P}(z)^{R}, q_{P}(z)\right)$. Again by Lemma $3.2, t_{R} b_{R} \rightarrow\{\infty\}^{\Delta_{R}}=$ $a_{R}(z)$, and thus $r_{t}^{(P)}(z) \rightarrow z$. The limiting behavior for $s_{t}^{(P)}$ is proved similarly. 


\section{Finite energy}

Let $\Phi^{+}$denote the positive $\mathbb{Q}$-roots of $G$ and let $\delta=1 / 2 \sum_{\lambda \in \Phi^{+}} \lambda$ with each root counted with multiplicity.

Definition 7.1. A piecewise-smooth map $f: M \rightarrow N$ between Riemannian manifolds (which may have finite quotient singularities) is said to have finite energy if

$$
E(f)=\int_{M}\left|d f_{z}\right|^{2} d V(z)<\infty,
$$

where the energy density $\mathcal{E}(f)(z)=\left|d f_{z}\right|^{2}$ is the norm squared of the differential $d f_{z}: T_{z} M \rightarrow T_{f(z)} N$. Define $f$ to have almost finite energy if for all $\epsilon>0$,

$$
\int_{M}\left|d f_{z}\right|^{2} e^{-\epsilon d_{M}\left(z, z_{0}\right)} d V(z)<\infty,
$$

where $z_{0} \in M$ is any fixed basepoint.

Remark 7.2. Say $M=N=\Gamma \backslash X$ and let $\left\{X_{P}\right\}_{P \in \mathcal{P}}$ be a tiling of $X$; it is not difficult to see that $f$ has almost finite energy if and only if for all $\epsilon>0$,

$$
\int_{\Gamma \backslash X}\left|d f_{z}\right|^{2} a(z)^{-\epsilon \delta} d V(z)<\infty,
$$

where $a(z)^{\delta}$ represents the function equal to $a_{P}(z)^{\delta}$ in $X_{P}\left(\right.$ and 1 in $\left.X_{0}\right)$.

Theorem 7.3. Let $r: X \rightarrow X_{0}$ be the $\Gamma$-invariant retraction onto the central tile of a tiling as in Theorem 6.1, and let $r^{\prime}$ be the induced retraction on $\Gamma \backslash X$. Assume $G$ is almost $\mathbb{Q}$-simple and that $\Gamma \backslash X$ is noncompact. Then $r^{\prime}$ has almost finite energy if and only if $G_{\mathbb{C}} \neq \mathrm{SL}(2, \mathbb{C})$. Furthermore, $r^{\prime}$ has finite energy if and only if $G_{\mathbb{C}}$ is not equal to $\mathrm{SL}(2, \mathbb{C}), \mathrm{SL}(2, \mathbb{C}) \times \mathrm{SL}(2, \mathbb{C}), \mathrm{SL}(3, \mathbb{C})$, or a $\mathbb{Q}$-split form of $\mathrm{SO}(5, \mathbb{C})$. The same assertions hold for the diffeomorphisms of Theorem 6.1.

Remark. In terms of $G$, the infinite energy cases are where $G$ is locally isomorphic to $\operatorname{SL}(2, \mathbb{R}), \operatorname{SL}(2, \mathbb{C})$, a non- $\mathbb{Q}$-split form of $\operatorname{SL}(2, \mathbb{R}) \times \operatorname{SL}(2, \mathbb{R})$ (the Hilbert modular surface case), $\mathrm{SL}(3, \mathbb{R}), \mathrm{SU}(2,1)$, or a $\mathbb{Q}$-split form of $\mathrm{SO}(3,2)$. In all these cases except for $\mathrm{SL}(2, \mathbb{R}), r^{\prime}$ has almost finite energy.

If $G$ is not almost $\mathbb{Q}$-simple, we may, by replacing $\Gamma$ with a subgroup of finite index, assume that $r^{\prime}: \Gamma \backslash X \rightarrow \Gamma \backslash X$ decomposes into a product with factors corresponding to the almost $\mathbb{Q}$-simple factors of $G$. Clearly this map has (almost) finite energy if and only if it does on each factor; thus we have the corollary:

Corollary 7.4. $r^{\prime}$ has (almost) finite energy if and only if none of the almost $\mathbb{Q}$-simple factors of $G$ which are $\mathbb{Q}$-isotropic have complexifications on the above lists. 
Proof of Theorem 7.3. We consider $r^{\prime}$; the situation for the diffeomorphism is similar. Let $P \in \mathcal{P}$. By Theorem 6.1, the restriction of $r$ to $X_{P} \cong A_{P}\left(b_{P}\right) \times e(P)$ corresponds to the retraction of the first factor onto $\left\{b_{P}\right\}$. By Borel's formula [10, $\S 4.3]$ for the metric on $X \cong A_{P} \times e(P)$, the energy density is thus ${ }^{5}$

$$
\mathcal{E}\left(\left.r\right|_{X_{P}}\right)(z) \sim \max _{\lambda \in \Phi^{+}} a_{P}(z)^{2 \lambda},
$$

where $g \sim h$ means that $C^{-1} h \leq g \leq C h$ for some constant $C>0$. On the other hand, the volume form corresponds $[10, \S 4.4]$ to

$$
d V_{X} \sim a_{P}(z)^{-2 \delta} \bigwedge_{\alpha \notin \Delta^{P}} \frac{d a_{P}^{\alpha}}{a_{P}^{\alpha}} \wedge d V_{e(P)} .
$$

Thus the energy in $\pi\left(X_{P}\right)$ (which is homeomorphic to $A_{P}\left(b_{P}\right) \times \Gamma_{P} \backslash e(P)$ by Proposition 2.2(iv)) is

$$
E\left(\left.r^{\prime}\right|_{\pi\left(X_{P}\right)}\right) \sim \max _{\lambda \in \Phi^{+}} \int_{A_{P}(1)} a^{2(\lambda-\delta)} d V_{A_{P}}(a)=\max _{\lambda \in \Phi^{+}} \prod_{\alpha \notin \Delta^{P}} \int_{1}^{\infty} a^{2 \alpha\left(\lambda-\delta, \beta_{\alpha}\right)} \frac{d a^{\alpha}}{a^{\alpha}} .
$$

This is finite for all $P$ (in other words, $r^{\prime}$ has finite energy) if and only if

$$
\text { for all } \lambda \in \Phi^{+},\left(\lambda-\delta, \beta_{\alpha}\right)<0 \text { for all } \alpha \in \Delta .
$$

By Remark 7.2, $r^{\prime}$ has almost finite energy if and only if the weaker condition

$$
\text { for all } \lambda \in \Phi^{+},\left(\lambda-\delta, \beta_{\alpha}\right) \leq 0 \text { for all } \alpha \in \Delta \text {. }
$$

is satisfied.

To determine when these conditions are not met, first assume the complexification $G_{\mathbb{C}}$ is almost simple and $\mathbb{Q}$-rank $G=\mathbb{C}$-rank $G$. Then (7.1) and (7.2) are assertions about the $\mathbb{C}$-root system $\Phi_{\mathbb{C}}$ of $G_{\mathbb{C}}$. The highest root and $\delta$ are enumerated for all simple root systems in [15, Planche I-IX] ( $\delta$ is denoted there as $\rho$ ); it follows easily that the only simple root systems failing (7.1) are $A_{1}, A_{2}$, and $B_{2}$, and the only one failing (7.2) is $A_{1}$.

Now say $\mathbb{Q}$-rank $G<\mathbb{C}$-rank $G$ (still assuming $G_{\mathbb{C}}$ is almost simple). Then by restriction, (7.1) is certainly implied by the corresponding assertion for $\Phi_{\mathbb{C}}$. If this fails (that is, for $\mathbb{C}$-root systems $A_{1}, A_{2}$, or $B_{2}$ ) then the only possibility is that $\mathbb{Q}$-rank $G=1$ and $\mathbb{C}$-rank $G=2$. (Since $\Gamma \backslash X$ is noncompact, $\mathbb{Q}$-rank $G>0$.) Let $\Delta_{\mathbb{C}}=\left\{\alpha, \alpha^{\prime}\right\}$ be the simple $\mathbb{C}$-roots (with $\alpha^{\prime}$ the shorter root in the case $B_{2}$ ) and denote the restriction from $\mathbb{C}$-roots to $\mathbb{Q}$-roots by an overbar. We will use the

5 Since for us $G$ acts on the left, $a$ in the formula in [10] should as usual be replaced by $a^{-1}$. 
classification theory [49, Table II] to determine the restriction. In the case $A_{2}$, the $\mathbb{Q}$-index must be ${ }^{2} A_{2,1}^{(1)}$, for which $\bar{\alpha}=\bar{\alpha}^{\prime}$. Thus $\Phi^{+}=\{\bar{\alpha}, 2 \bar{\alpha}\}$ and $\delta=2 \bar{\alpha}$, so (7.1) fails though (7.2) is satisfied for the non- $\mathbb{Q}$-split forms of $\mathrm{SL}(3, \mathbb{C})$. In the case $B_{2}$, the $\mathbb{Q}$-index must be $B_{2,1}$, for which $\bar{\alpha}^{\prime}=0$. Thus $\Phi^{+}=\{\bar{\alpha}\}$ and $\delta=3 / 2 \bar{\alpha}$, so (7.1) is satisfied for the non- $\mathbb{Q}$-split forms of $\mathrm{SO}(5, \mathbb{C})$.

Finally, say $G_{\mathbb{C}}$ is not almost simple. In this case, $G$ is obtained (up to isogeny) by the restriction of scalars $R_{k / \mathbb{Q}} G^{\prime}$, where $G^{\prime}$ is defined over a finite extension $k$ of $\mathbb{Q}$ and $G_{\mathbb{C}}^{\prime}$ is almost simple. The $\mathbb{Q}$-root system of $G$ is identical with the $k$-root system of $G^{\prime}$, except that the multiplicities, and hence $\delta$, are multiplied by $[k: \mathbb{Q}]>1$. Thus if $(7.2)$ is satisfied for $G^{\prime},(7.1)$ will be satisfied for $G$ (since $\left.\left(\delta, \beta_{\alpha}\right)>0\right)$. The previous argument shows that $(7.2)$ for $G^{\prime}$ will fail only if $G_{\mathbb{C}}^{\prime}$ has type $A_{1}$. In this case, $\delta=[k: \mathbb{Q}](\alpha / 2)$ where $\alpha$ is the unique simple $\mathbb{Q}$-root, so (7.1) fails only if $[k: \mathbb{Q}]=2$ (and then (7.2) is satisfied). This is the case $G_{\mathbb{C}}=\mathrm{SL}(2, \mathbb{C}) \times \mathrm{SL}(2, \mathbb{C})$.

\section{Neighborhoods of boundary faces}

Another application of tilings is to give an explicit description of a cofinal system of $\Gamma$-invariant neighborhoods of each closed boundary face $\overline{e(R)}$ of $\bar{X}$. Namely, let $\left\{\bar{X}_{P}\right\}_{P \in \mathcal{P}}$ be a $\Gamma$-invariant tiling of $\bar{X}$ with parameter $\mathbf{b}$, and for all $R \in \mathcal{P}$, define

$$
\bar{U}_{R}=\coprod_{P \subseteq R} \bar{X}_{P}
$$

\section{Theorem 8.1.}

(i) $\bar{U}_{R}$ is an open $\Gamma_{R}$-invariant neighborhood of $\overline{e(R)}$, stable under the geodesic action of $\bar{A}_{R}(1)$.

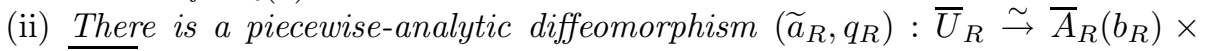
$\overline{e(R)}$, where $\widetilde{a}_{R}$ is determined by the equations

$$
\left(b_{R}^{-1} \widetilde{a}_{R}(z)\right)^{\alpha}=\left(b_{P}^{-1} a_{P}(z)\right)^{\alpha} \quad \text { for } z \in \bar{X}_{P}, P \subseteq R \text {, and } \alpha \in \Delta_{P} \backslash \Delta_{P}^{R} .
$$

This diffeomorphism is $A_{R}(1) \times \Gamma_{R}$-equivariant.

(iii) The natural projection $\Gamma_{R} \backslash \bar{U}_{R} \rightarrow \pi\left(\bar{U}_{R}\right)$ is a homeomorphism.

(iv) Let $\bar{U}_{R, t}$ be the neighborhoods corresponding to the family of tilings with parameters $t \cdot \mathbf{b}, t \in \mathrm{cl}(A(1))$. As $t$ tends to infinity under the action of a dominant 1-parameter subgroup such that $t^{\alpha} \rightarrow \infty$ for all $\alpha \notin \Delta^{R}$, the open set $\bar{U}_{R, t}$ shrinks and runs over a cofinal system of $\Gamma_{R}$-invariant neighborhoods of $\overline{e(R)}$. 
Remark. (1) Except in $\bar{X}_{R}$, the function $\widetilde{a}_{R}$ in (ii) is not in general constant on the orbits of ${ }^{0} R$. Thus the diffeomorphism $\left(\widetilde{a}_{R}, q_{R}\right)$ is not ${ }^{0} R$-equivariant and the induced sections of $q_{R}: \bar{U}_{R} \rightarrow \overline{e(R)}$ are not canonical cross-sections of $q_{R}$.

(2) Previously Zucker [52] constructed neighborhoods of the boundary faces of $\Gamma \backslash \bar{X}$ which correspond to smoothed versions of our $\pi\left(\bar{U}_{R}\right)$.

Example. Assume $X$ is hermitian symmetric (that is, a bounded symmetric domain) and (for simplicity) $G$ is almost $\mathbb{Q}$-simple. For $Q$ a maximal parabolic, there is a decomposition $X \cong F \times C \times N_{Q}$, where $F$ is a hermitian symmetric space of lower rank, $C$ is a self-adjoint homogeneous cone, and $N_{Q}$ is the unipotent radical of $Q$. The geodesic action of $A_{Q}$ corresponds to the dilation on $C$, thus $e(Q) \cong F \times\left(A_{Q} \backslash C\right) \times N_{Q}$. Now restrict attention to $B \subset F$, a small ball neighborhood of some $y \in F$ which is "away from the ends" (that is, in the central tile of the induced tiling). Then it is not difficult to see that $\bar{U}_{Q} \cap\left(B \times C \times N_{Q}\right) \cong B \times C_{0} \times N_{Q}$, where $C_{0}$ is the "adapted core" constructed in [43, §4]. Hence by [4, III, §6.11] we obtain a cofinal family of neighborhoods in the "Satake topology" after taking quotient by $\Gamma$. This illustrates Zucker's result [51] that the Baily-Borel-Satake compactification $\Gamma \backslash X^{*}$ may be realized as a topological quotient of $\Gamma \backslash \bar{X}$. Note too, that the explicit nature of the normal vector to the boundary of these neighborhoods (see (ii) above) was used in a crucial way in [43, $\S \S 4.1,9.7]$.

Proof of Theorem 8.1. Let $Y(R)$ be the open neighborhood $\bigcup_{P \subseteq R} X(P)$ of $\overline{e(R)}$ in $\bar{X}$. The projection map $q_{R}: X(R) \rightarrow e(R)$ extends to $q_{R}: Y(R) \rightarrow \overline{e(R)}$, and we wish to construct a trivialization of this bundle. (The canonical trivialization $X(R) \cong \bar{A}_{R} \times e(R)$ will not do, since it does not in general extend to any $X(P)$ for $P \subsetneq R$.)

A trivialization of $q_{R}$ on $X(P)$ for each $P \subseteq R$ is constructed in [12, §5.4(7)]. Namely decompose $[12, \S 4.3]$

$$
A_{P} \cong\left(\mathbb{R}^{>0}\right)^{\Delta_{P}} \cong\left(\mathbb{R}^{>0}\right)^{\Delta_{P} \backslash \Delta_{P}^{R}} \times\left(\mathbb{R}^{>0}\right)^{\Delta_{P}^{R}} \equiv A_{R} \times A_{P, R} ;
$$

this clearly extends ${ }^{6}$ to $\bar{A}_{P} \cong \bar{A}_{R} \times \bar{A}_{P, R}$. Let $a_{R}^{(P)}(z)$ denote the projection of $a_{P}(z) \in \bar{A}_{P}$ to $\bar{A}_{R}$ with respect to this decomposition. Then

$$
\left(a_{R}^{(P)}, q_{R}\right): X(P) \cong \bar{A}_{R} \times e(R)(P)
$$

is the trivialization, where $e(R)(P) \equiv \coprod_{P \subset S \subset R} e(S)$.

To obtain a piecewise-analytic trivialization on all of $Y(R)$, we piece together the above trivializations using a tiling: lift the tiling $\left\{\bar{X}_{P} \cap \overline{e(R)}\right\}_{P \subseteq R}$ of $\overline{e(R)}$ to

\footnotetext{
6 Note that in general $A_{P, R} \neq A_{P}^{R}$ and that (1.1) does not in general extend to a decomposition of $\bar{A}_{P}[52,(1.3)]$.
} 
the tiling $\left\{q_{R}^{-1}\left(\bar{X}_{P}\right)\right\}_{P \subseteq R}$ of $Y(R)$ and define $\widetilde{a}_{R}$ by the equations

$$
b_{R}^{-1} \widetilde{a}_{R}(z) \equiv b_{R}^{(P)-1} a_{R}^{(P)}(z) \quad \text { for } z \in q_{R}^{-1}\left(\bar{X}_{P}\right), P \subseteq R,
$$

where $b_{R}^{(P)}$ is the projection of $b_{P}$ to the first factor of (8.1) - this agrees with the definition in (ii). It is easy to check that $\widetilde{a}_{R}$ is piecewise-analytic and $\Gamma_{R}$-invariant. Then $\left(\widetilde{a}_{R}, q_{R}\right): Y(R) \stackrel{\sim}{\rightarrow} \bar{A}_{R} \times \overline{e(R)}$ is the desired trivialization.

In order now to prove (i) and (ii), we simply note that

$$
\bar{X}_{P}=\bar{A}_{R}(1) \mathbf{o}\left(\bar{A}_{P, R}(1) \mathbf{o} \partial^{P} X_{0}\right)
$$

and thus that $\bar{U}_{R}=\left\{z \in Y(R) \mid \tilde{a}_{R}(z) \in \bar{A}_{R}\left(b_{R}\right)\right\}$. For (iii), consider $\gamma \in \Gamma$ such that $\gamma \cdot \bar{U}_{R} \cap \bar{U}_{R} \neq \emptyset$. Then by $\Gamma$-invariance of the tiling, there exists $P \subseteq R$ such that ${ }^{\gamma} P \subseteq R$, or $P \subseteq R \cap R^{\gamma}$. Thus we must have $R=R^{\gamma}$, that is, $\gamma \in \Gamma_{R}$.

For (iv), we first single out a lemma which is of independent interest:

Lemma 8.2. Let $t_{2} \in \operatorname{cl}\left(A\left(t_{1}\right)\right)$. Then $\bar{X}_{S, t_{2}} \subseteq \bigcup_{P \subseteq S} \bar{X}_{P, t_{1}}$.

Proof of the lemma. Fix $z \in \bar{X}_{S, t_{2}}$. As $t$ passes from $t_{2}$ to $t_{1}$, the first factor of $\bar{X}_{S, t} \cong \bar{A}_{S}\left(t_{S} b_{S}\right) \times q_{S}\left(\bar{X}_{S, t}\right)$ is nondecreasing, so the only way $z$ can fail to belong to $\bar{X}_{S, t}$ is because of the second factor; it follows from Proposition 2.2(ii) in this case that $z \in \bar{X}_{P, t}$ for some $P \subsetneq S$ and some $t$ and one can use induction on $\mathcal{P}$-rank $S$.

Now let $t$ tend to infinity as in (iv). By the lemma, the sets $\operatorname{cl}\left(\bar{U}_{R, t}\right)$ are nonincreasing. The lemma also implies that $z \in \bigcap_{t} \bar{U}_{R, t}$ must belong to some $\bar{X}_{P, t}$ for all $t$ sufficiently large, where $P \subseteq R$ is fixed, and thus $a_{P}(z) \in \bar{A}_{P}\left(t_{P} b_{P}\right)$. Therefore $\left(b_{P}^{-1} a_{P}(z)\right)^{\alpha}>t_{P}^{\alpha}=t^{\alpha}\left(t^{P}\right)^{-\alpha} \rightarrow \infty$ for all $\alpha \notin \Delta^{R}$ (note that $\left(t^{P}\right)^{-\alpha}$ is bounded from below by Remark 3.3(i)), and so $z \in \overline{e(R)}$. Thus $\pi\left(\operatorname{cl}\left(\bar{U}_{R, t}\right)\right)$ is a decreasing family of compact sets with intersection $\pi(\overline{e(R)})$ and therefore (compare $[12, \S 10.2])$ any open neighborhood of $\pi(\overline{e(R)})$ must contain one of them.

\section{The space of regular parameters}

As indicated in the introduction, it is also of interest to study tilings whose parameters are not necessarily large. In this final section we make a first step in this direction. Define a parameter $\mathbf{b} \in \mathcal{B}$ to be regular if a tiling exists with parameter $\mathbf{b}$, and denote the subset of regular parameters by $\mathcal{B}_{\text {reg }}$; by Proposition 2.6 this set is preserved under automorphisms of $G$ defined over $\mathbb{Q}$. Theorem 5.7 demonstrated that the set of regular $\Gamma$-invariant tilings $\mathcal{B}_{\text {reg }}^{\Gamma}$ is nonempty by constructing an analytic tiling for large $\Gamma$-invariant parameters. We will now show in Theorem 9.6 that $\mathcal{B}_{\text {reg }}^{\Gamma}$ is an open subset of $\mathcal{B}^{\Gamma}$ and is $\operatorname{cl}(A(1))$-invariant. We also find that 
any $\Gamma$-invariant tiling is analytic and that any $\operatorname{cl}(A(1))$-family of such tilings is $\mathcal{W}$-adapted for some $\Gamma$-invariant cylindrical cover $\mathcal{W}$. For large parameters this was part of Theorem 5.7 and was the our main tool in using tilings. Along the way we will give a criterion for when a parameter is regular, which may be of use in studying degenerations of tilings.

Proposition 9.1. Fix $\mathbf{b} \in \mathcal{B}^{\Gamma}$ and let a subset $\bar{X}_{R} \subseteq \bar{X}$ be given for each $R \in \mathcal{P}$. Then $\left\{\bar{X}_{R}\right\}_{R \in \mathcal{P}}$ is a tiling of $\bar{X}$ with parameter $\mathbf{b}$ if and only if there exists a $\Gamma$-invariant cylindrical cover $\mathcal{W}=\left\{\bar{W}_{P}\right\}_{P \in \mathcal{P}}$ of $\bar{X}$ such that for all $P, R \in \mathcal{P}$,

(i) $b_{P} \sim \bar{W}_{P}$, and

(ii) $\bar{X}_{R} \cap \bar{W}_{P}= \begin{cases}X(P)_{R} \cap \bar{W}_{P} & \text { for } P \subseteq R, \\ \emptyset & \text { for } P \nsubseteq R .\end{cases}$

Before beginning the proof we single out a simple lemma which will be useful later as well.

Lemma 9.2. Let $\bar{K}_{P}$ be the closure of a cylindrical set relative to $P$. The cylindrical sets $\bar{W}_{P} \supset \bar{K}_{P}$ are cofinal among $\Gamma_{P}$-invariant neighborhoods of $\bar{K}_{P}$.

Proof. Project to $\Gamma_{P} \backslash X(P) \cong \bar{A}_{P} \times \Gamma_{P} \backslash e(P)$. The set $\bar{K}_{P}$ (resp. $\bar{W}_{P}$ ) projects to a product of compact (resp. relatively compact) sets with the $\Gamma_{P} \backslash e(P)$ factor having full unipotent fibers. (Recall that $\Gamma_{P} \backslash e(P)$ is fibered by $\left(\Gamma_{P} \cap N_{P}\right) \backslash N_{P}$ over a locally symmetric space for a Levi $\mathbb{Q}$-subgroup for ${ }^{0} P$.) The result follows.

Proof of Proposition 9.1. First we assume (i) and (ii) hold and demonstrate that $\left\{\bar{X}_{R}\right\}_{R \in \mathcal{P}}$ is a tiling. By Proposition 2.2(ii), $\partial^{R} X(P)_{0}=X(P)_{0} \cap \operatorname{cl}\left(X(P)_{R}\right)$. So for $R \in \mathcal{P}$ define $\partial^{R} X_{0} \equiv X_{0} \cap \operatorname{cl}\left(\bar{X}_{R}\right)$. Then it follows from (ii) that

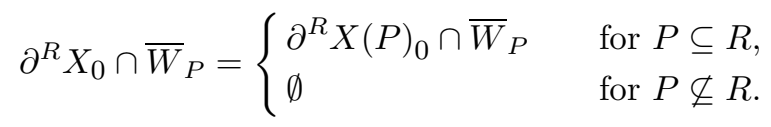

Now (ii), (9.1) and Lemma 5.4 imply that $\bar{X}_{R} \cap \bar{W}_{P}=\bar{A}_{R}(1) \mathbf{o}\left(\partial^{R} X_{0} \cap \bar{W}_{P}\right)$ for $P \subseteq R$, which yields

$$
\bar{X}_{R}=\bar{A}_{R}(1) \mathbf{o} \partial^{R} X_{0}
$$

(since by (ii) and (9.1) every point in $\bar{X}_{R}$ or $\partial^{R} X_{0}$ belongs to $\bar{W}_{P}$ for some $P \subseteq R$ ). The conditions of Definition 2.1 clearly follow from (ii), (9.1), and (9.2).

Now assume that $\left\{\bar{X}_{R}\right\}_{R \in \mathcal{P}}$ is a tiling ( $\Gamma$-invariant since $\mathbf{b}$ is). We will construct the desired $\Gamma$-invariant cylindrical cover $\mathcal{W}=\left\{\bar{W}_{P}\right\}_{P \in \mathcal{P}}$ by a modification of the inductive argument in $[52,(3.6)]$. Namely, totally order the $\Gamma$-conjugacy classes of parabolics $[P]$ such that $\mathcal{P}$-rank $P<\mathcal{P}$-rank $P^{\prime}$ implies that $[P]<\left[P^{\prime}\right]$. Then given a $\Gamma$-conjugacy class $[P]$, assume $\bar{W}_{P^{\prime}}$ has been constructed for $P^{\prime}$ in all higher $\Gamma$-conjugacy classes. Set 


$$
\bar{K}_{P}=\operatorname{cl}\left(\bar{A}_{P}\left(b_{P}\right)\right) \times\left(e(P) \backslash \bigcup_{P^{\prime} \subsetneq P} q_{P}\left(\bar{W}_{P^{\prime}}\right)\right) .
$$

and define $\bar{W}_{P}=\bar{A}_{P}\left(s_{P}\right) \times O_{P}$ to be a product neighborhood of $\bar{K}_{P}$. If the previously constructed collection $\left\{\bar{W}_{P^{\prime}}\right\}_{\left[P^{\prime}\right]>[P]}$ is $\Gamma$-invariant, we see that $\bar{K}_{\gamma P}=$ $\gamma \cdot \bar{K}_{P}$, and thus we can assume $\bar{W}_{\gamma P}=\gamma \cdot \bar{W}_{P}$. We will prove below the claim that $\bar{K}_{P}$ lies in the complement of $\bigcup_{R \nsupseteq P} \operatorname{cl}\left(\bar{X}_{R}\right)$. Then since this latter set is $\Gamma_{P^{-}}$ invariant, $\bar{W}_{P}$ (or even $\mathrm{cl}\left(\bar{W}_{P}\right)$ ) may likewise be chosen to be in its complement by Lemma 9.2. If we continue in this fashion, we will have constructed $\mathcal{W}$ satisfying (i) and the second line of (ii). For the first line of (ii) one needs to check that the decompositions $\left\{\bar{X}_{R} \cap \bar{W}_{P}\right\}_{R \supseteq P}$ and $\left\{X(P)_{R} \cap \bar{W}_{P}\right\}_{R \supseteq P}$ of $\bar{W}_{P}$ agree, which follows from Proposition 2.4.

It remains to prove the claim. Note that the $\bar{W}_{P^{\prime}}$ satisfy by induction

$$
\bigcup_{P^{\prime} \subsetneq P} \bar{W}_{P^{\prime}} \supseteq \bigcup_{P^{\prime} \subsetneq P} \operatorname{cl}\left(\bar{X}_{P^{\prime}}\right)
$$

It follows that $\bar{K}_{P} \subseteq \operatorname{cl}\left(\bar{X}_{P}\right)$, which is disjoint from $\operatorname{cl}\left(\bar{X}_{R}\right)$ by Proposition 2.2(ii) unless $R \cap P \in \mathcal{P}$. But in this case the intersection is contained in $\operatorname{cl}\left(\bar{X}_{R \cap P}\right)$. Since $R \nsupseteq P, R \cap P \subsetneq P$ and so this is contained in $\bigcup_{P^{\prime} \subsetneq P} \bar{W}_{P^{\prime}}$. But such points have been removed from $\bar{K}_{P}$ by definition.

We can now characterize via cylindrical covers those parameters $\mathbf{b} \in \mathcal{B}^{\Gamma}$ for which a tiling exists. Recall that given $\mathbf{b}$ we defined normalized functions $\hat{a}_{P}=$ $a_{P} / b_{P}$.

Proposition 9.3. A tiling of $\bar{X}$ with parameter $\mathbf{b} \in \mathcal{B}^{\Gamma}$ exists if and only if there exists a $\Gamma$-invariant cylindrical cover $\mathcal{W}=\left\{\bar{W}_{P}\right\}_{P \in \mathcal{P}}$ of $\bar{X}$ such that for all $P$, $P^{\prime} \in \mathcal{P}$,

(i) $b_{P} \sim \bar{W}_{P}$, and

(ii) ${ }^{\prime} \hat{a}_{P}\left(\bar{W}_{P} \cap \bar{W}_{P^{\prime}}\right)^{P \vee P^{\prime}}$ lies in the central tile of $A_{P}^{P \vee P^{\prime}}$.

In this case, the tiling will be $\mathcal{W}$-adapted.

Proof. If a tiling $\left\{\bar{X}_{R}\right\}_{R \in \mathcal{P}}$ exists with parameter $\mathbf{b} \in \mathcal{B}^{\Gamma}$, Proposition 9.1 implies there exists a $\Gamma$-invariant distinguished cover $\mathcal{W}$ for which it is $\mathcal{W}$-adapted. In particular,

$$
\bar{X}_{R}=\bigcup_{P \subseteq R} X(P)_{R} \cap \bar{W}_{P} .
$$

Conversely, given a $\Gamma$-invariant cylindrical cover $\mathcal{W}$ satisfying (i), we may use (9.4) to define $\bar{X}_{R}$. Then Proposition 9.1 shows that this is a tiling if (ii) is satisfied. Thus it suffices to show that (ii) is equivalent to (ii) ${ }^{\prime}$ given that $\bar{X}_{R}$ is defined as in (9.4). 
Now (ii) is easily seen to be equivalent (given (9.4)) to

$$
X(P)_{R} \cap \bar{W}_{P} \cap \bar{W}_{P^{\prime}}= \begin{cases}X\left(P^{\prime}\right)_{R} \cap \bar{W}_{P} \cap \bar{W}_{P^{\prime}} & \text { for } P, P^{\prime} \subseteq R, \\ \emptyset & \text { for } P \subseteq R, P^{\prime} \nsubseteq R .\end{cases}
$$

We claim this is equivalent to

$$
\bar{W}_{P} \cap \bar{W}_{P^{\prime}} \subseteq \coprod_{R \supseteq P \vee P^{\prime}} X(P)_{R} \quad \text { for } P, P^{\prime} \in \mathcal{P} \text {. }
$$

For it is clear that the second line of (9.5) is equivalent to (9.6). Now (9.6) implies that $\left\{X(P)_{R} \cap \bar{W}_{P} \cap \bar{W}_{P^{\prime}}\right\}_{R \supseteq P \vee P^{\prime}}$ is a decomposition of $\bar{W}_{P} \cap \bar{W}_{P^{\prime}}$ satisfying the conditions of Proposition 2.4. Since the same holds true with $P$ and $P^{\prime}$ interchanged, these two tilings must agree by that proposition; in other words, the first line of (9.5) holds. This proves the claim.

Now assume (9.6) holds. The left-hand side is $\bar{A}_{P \vee P^{\prime}}(1)$-invariant and therefore contains its projection to $e\left(P \vee P^{\prime}\right)$. But only the $R=P \vee P^{\prime}$ factor on the righthand side intersects $e\left(P \vee P^{\prime}\right)$. Thus $q_{P \vee P^{\prime}}\left(W_{P} \cap W_{P^{\prime}}\right)$ lies in $e\left(P \vee P^{\prime}\right) \cap X(P)_{P \vee P^{\prime}}$. The application of $\hat{a}_{P}$ now yields (ii)' . (Note that we use the identification of $\hat{a}_{P}\left(e\left(P \vee P^{\prime}\right)\right)$ with $A_{P}^{P \vee P^{\prime}}$ as in the proof of Proposition 3.7.)

On the other hand, (ii) and Lemma 3.5 imply that $\hat{a}_{P}\left(W_{P} \cap W_{P^{\prime}}\right)^{P \vee P^{\prime}} \subseteq$ $\left\langle\bar{A}_{P}\right\rangle_{P \vee P^{\prime}}^{P \vee P^{\prime}}=\partial^{P \vee P^{\prime}}\left\langle A_{P}\right\rangle_{0}$. We find then that

$$
\hat{a}_{P}\left(\bar{W}_{P} \cap \bar{W}_{P^{\prime}}\right) \subseteq \bar{A}_{P \vee P^{\prime}} \cdot \partial^{P \vee P^{\prime}}\left\langle A_{P}\right\rangle_{0} \subseteq \coprod_{R \supseteq P \vee P^{\prime}}\left\langle\bar{A}_{P}\right\rangle_{R}
$$

where we use Lemma 3.5 again for the last inclusion. This implies (9.6) since $\bar{W}_{P} \cap \bar{W}_{P^{\prime}}$ is ${ }^{0} P$-invariant.

To prove our final theorem, we will need the above cylindrical covers to have as few nonempty intersections as possible.

Definition 9.4 (compare with $[52,(3.6)]^{7}$ ). A cylindrical cover $\mathcal{W}$ of $X$ is said to be distinguished if

$$
W_{P} \cap W_{P^{\prime}}=\emptyset \text { for } P \nsubseteq P^{\prime} \text { and } P \nsupseteq P^{\prime}
$$

A $\Gamma$-invariant cylindrical cover $\mathcal{W}$ is $\Gamma$-distinguished if

$$
W_{P} \cap W_{P^{\prime}}=\emptyset \text { for } P \npreceq P^{\prime} \text { and } P \nsucceq P^{\prime} \text {. }
$$

\footnotetext{
${ }^{7}$ Our notion of $\Gamma$-distinguished corresponds, after taking quotient by $\Gamma$, to what is called distinguished in [52]. Our notion of distinguished, on the other hand, appears to be new.
} 
(We write $P \preccurlyeq P^{\prime}$ to mean that ${ }^{\gamma} P \subseteq P^{\prime}$ for some $\gamma \in \Gamma$.)

Remark. A $\Gamma$-invariant distinguished cover is clearly $\Gamma$-distinguished. On the other hand, it is not difficult to show that a $\Gamma$-distinguished cover $\mathcal{W}$ is distinguished if and only if

$$
\Pi\left(\bigcup_{P \subseteq R} W_{P}\right)=\Gamma_{R} \quad \text { for all } R \in \mathcal{P}
$$

where

$$
\Pi(K) \equiv\{\gamma \in \Gamma \mid \gamma K \cap K \neq \emptyset\} .
$$

In [27, Theorem 1.3.2] and [52, (3.6)], $\Gamma$-distinguished covers are constructed such that $\Pi\left(W_{R}\right)=\Gamma_{R}$ for $R \in \mathcal{P}$; a priori this is weaker than (9.9). By using the existence of $\Gamma$-invariant tilings, however, we can show that distinguished covers exist:

Proposition 9.5. The $\Gamma$-invariant cylindrical covers in Propositions 9.1 and 9.3 may be chosen to be distinguished.

Proof. Note that in the inductive construction of $\bar{W}_{P}$ for Proposition 9.1, we arranged that $\bar{K}_{P} \subseteq \operatorname{cl}\left(\bar{X}_{P}\right)$ and that for $\left[P^{\prime}\right]>[P], \operatorname{cl}\left(\bar{W}_{P^{\prime}}\right)$ lies in the complement of $\bigcup_{R \nsupseteq P^{\prime}} \mathrm{cl}\left(\bar{X}_{R}\right)$. Thus $\bar{K}_{P}$ is in the complement of

$$
\bigcup_{P^{\prime} \not P,\left[P^{\prime}\right]>[P]} \operatorname{cl}\left(\bar{W}_{P^{\prime}}\right)
$$

Since this set is $\Gamma_{P}$-invariant, the neighborhood $\bar{W}_{P}$ may likewise be chosen in its complement by Lemma 9.2. This establishes (9.7) in the case $\left[P^{\prime}\right]>[P]$. For the case $\left[P^{\prime}\right]=[P]$, we need to choose $\bar{W}_{P}$ such that $\bar{W}_{P} \cap \bar{W}_{\gamma_{P}}=\emptyset$ for $\gamma \notin \Gamma_{P}$, that is, such that $\Pi\left(\bar{W}_{P}\right) \subseteq \Gamma_{P}$. However $\bar{K}_{P} \cap \bar{K}_{\gamma_{P}} \subseteq \operatorname{cl}\left(\bar{X}_{P}\right) \cap \operatorname{cl}\left(\bar{X}_{\gamma_{P}}\right)=\emptyset$ by Proposition 2.2(ii), so $\Pi\left(\bar{K}_{P}\right) \subseteq \Gamma_{P}$. Now (compare [12, §10.3]) let $C \subseteq \bar{K}_{P}$ be compact such that $\bar{K}_{P}=\Gamma_{P} \cdot \bar{C}$ and let $U$ be a relatively compact neighborhood of $C$. Since $\Gamma$ acts properly on $\bar{X}[12, \S 9.3]$, the sets $\Pi(C) \subseteq \Pi(U)$ are finite $[14$, III, $\S 4.5]$. By shrinking $U$ if necessary, we can assume that $\Pi(C)=\Pi(U)$. Thus if we choose $\bar{W}_{P}$ so that $\bar{K}_{P} \subseteq \bar{W}_{P} \subseteq \Gamma_{P} \cdot U$, we have $\Pi\left(\bar{W}_{P}\right) \subseteq \Gamma_{P} \cdot \Pi(U) \cdot \Gamma_{P}=$ $\Gamma_{P} \cdot \Pi(C) \cdot \Gamma_{P} \subseteq \Gamma_{P}$ as desired.

Theorem 9.6. The subset $\mathcal{B}_{\text {reg }}^{\Gamma} \subseteq \mathcal{B}^{\Gamma}$ of regular $\Gamma$-invariant parameters is open and $\operatorname{cl}(A(1))$-invariant. For any $\mathbf{b} \in \mathcal{B}_{\text {reg }}^{\Gamma}$, there exists a $\Gamma$-invariant distinguished cover $\mathcal{W}$ and a neighborhood $U$ of $\mathbf{b}$ for which the tilings of $\bar{X}$ with parameters $t \cdot \mathbf{b}^{\prime}, t \in \operatorname{cl}(A(1))$ and $\mathbf{b}^{\prime} \in U$, are all $\mathcal{W}$-adapted. All tilings of $\bar{X}$ are analytic and depend analytically on $\mathbf{b}$. 
Proof. By Propositions 9.3 and 9.5, a parameter $\mathbf{b} \in \mathcal{B}^{\Gamma}$ belongs to $\mathcal{B}_{\mathrm{reg}}^{\Gamma}$ if and only if there exists a $\Gamma$-invariant distinguished cover $\mathcal{W}$ satisfying (i) and (ii)', and in this case the tiling is $\mathcal{W}$-adapted. Since the number of $\Gamma$-conjugacy classes of parabolics is finite, condition (i) is clearly an open condition. Clearly we may shrink $\mathcal{W}$ and replace (ii) ${ }^{\prime}$ by

(ii) $)^{\prime \prime} \hat{a}_{P}\left(\operatorname{cl}\left(\bar{W}_{P}\right) \cap \operatorname{cl}\left(\bar{W}_{P^{\prime}}\right)\right)^{P \vee P^{\prime}}$ lies in the interior of the central tile of $A_{P}^{P \vee P^{\prime}}$.

The left-hand set of (ii)" is compact (since $\beta_{\alpha}$ for $\alpha \in \Delta_{P}^{P \vee P^{\prime}}$ is bounded from above on the central tile and from below on $\left.\hat{a}_{P}\left(\operatorname{cl}\left(\bar{W}_{P}\right)\right)^{P \vee P^{\prime}}\right)$ and depends continuously on $\mathbf{b}$. We would like to conclude that requiring (ii)" for all $P, P^{\prime} \in \mathcal{P}$ is an open condition on $\mathbf{b}$, but unfortunately the number of $\Gamma$-conjugacy classes of pairs of parabolics (and hence independent conditions in (ii)") is not necessarily finite.

However it does suffice by $\Gamma$-invariance to restrict $P$ to belong to a finite set of representatives of $\Gamma$-conjugacy classes. Then if $P^{\prime} \subseteq P$ we may assume by an application of an element of $\Gamma_{P}$ that $P^{\prime}$ belongs to a finite set of representatives of $\Gamma_{P}$-conjugacy classes of parabolics in $P$. If on the other hand $P^{\prime} \nsubseteq P$, we may by the disjointness property (9.7) of a distinguished cover restrict $P^{\prime}$ to the finite set of parabolics containing $P$. Thus we obtain an open condition. Consequently (ii)" remains valid for $\mathbf{b}^{\prime}$ in a small neighborhood of $\mathbf{b}$, and so $\mathbf{b}^{\prime} \in \mathcal{B}_{\text {reg }}^{\Gamma}$.

If one replaces $\mathbf{b}$ by $t \cdot \mathbf{b}$ for $t \in \operatorname{cl}(A(1))$, the function $\hat{a}_{P}^{P \vee P^{\prime}}$ from (ii)" becomes multiplied by $\left(t_{P}^{P \vee P^{\prime}}\right)^{-1}$. This value belongs to the central tile of $A_{P}^{P \vee P^{\prime}}$ by Lemma 3.2, so condition (ii)" remains valid. Clearly (i) remains true, and consequently $\mathcal{B}_{\text {reg }}^{\Gamma}$ is $\operatorname{cl}(A(1))$-invariant.

The final statements are a consequence of adaptedness and the analyticity of the tiling asssociated to each parabolic (Proposition 4.1).

\section{References}

[1] J. Arthur, A trace formula for reductive groups I: Terms associated to classes in $G(\mathbb{Q})$, Duke Math. J. 45 (1978), 911-952.

[2] A. Ash, Small-dimensional classifying spaces for arithmetic subgroups of general linear groups, Duke Math. J. 51 (1984), 459-468.

[3] A. Ash and M. McConnell, Cohomology at infinity and the well-rounded retract for general linear groups, Duke Math. J. 90 (1997).

[4] A. Ash, D. Mumford, M. Rapoport, and Y. Tai, Smooth Compactifications of Locally Symmetric Varieties, Math. Sci. Press, Brookline 1975.

[5] M. Atiyah and R. Bott, The Yang-Mills equation over Riemann surfaces, Philos. Trans. Roy. Soc. London, Ser. A 308 (1983), 523-615.

[6] W. Baily and A. Borel, Compactification of arithmetic quotients of bounded symmetric domains, Ann. of Math. 84 (1966), 442-528.

[7] W. Ballmann, Nonpositively curved manifolds of higher rank, Ann. of Math. 122 (1985), 597-609.

[8] W. Ballmann and P. Eberlein, Fundamental groups of manifolds of nonpositive curvature, J. Diff. Geom. 25 (1987), 1-22. 
[9] A. Borel, Introduction aux groupes arithmétiques, Act. Sci. Ind. 1341, Hermann éd., Paris 1969.

[10] A. Borel, Stable real cohomology of arithmetic groups, Ann. Sci. ENS 7 (1974), 235-272.

[11] A. Borel, Stable real cohomology of arithmetic groups II, Manifolds and Lie groups. Papers in honor of Yozô Matsushima (J. Hano, et. al., eds.), Progress in Mathematics 14, Birkhäuser, Boston 1981, pp. 21-55.

[12] A. Borel and J-P. Serre, Corners and arithmetic groups, Comment. Math. Helv. 48 (1973), 436-491.

[13] A. Borel and N. Wallach, Continuous cohomology, discrete subgroups, and representation of reductive groups, Ann. of Math. Stud. 94, Princeton University Press, Princeton 1980.

[14] N. Bourbaki, General Topology, Part 1, Elements of Mathematics, Addison-Wesley, Reading 1966.

[15] N. Bourbaki, Groupes et algèbres de Lie, chapitres 4, 5 et 6, Éléments de mathématique, Masson, Paris 1981.

[16] K. Burns and R. Spatzier, Manifolds of nonpositive curvature and their buildings, Publ. Math. IHES 65 (1987), 35-59.

[17] K. Corlette, Archimedean superrigidity and hyperbolic geometry, Ann. of Math. 135 (1992), $165-182$.

[18] P. Eberlein and J. Heber, A differential geometric characterization of symmetric spaces of higher rank, Publ. Math. IHES 71 (1990), 33-44.

[19] H. Garland and M. S. Raghunathan, Fundamental domains for ( $\mathbb{R}-$ )rank 1 semi-simple Lie groups, Ann. of Math. 92 (1970), 279-326.

[20] M. Goresky, G. Harder, and R. MacPherson, Weighted Cohomology, Inv. Math. 116 (1994), 139-213.

[21] M. Goresky and R. MacPherson, Weighted cohomology of Satake compactifications, preliminary announcement (1988).

[22] D. Grayson, Reduction theory using semistability, I, Comment. Math. Helv. 59 (1984), $600-634$.

[23] D. Grayson, Reduction theory using semistability, II, Comment. Math. Helv. 61 (1986), 661-676.

[24] D. Grayson, Finite Generation of $K$-groups of a curve over a finite field [after Daniel Quillen], Algebraic K-theory, Proceedings, Oberwolfach 1980, Part I, Lecture Notes in Math. 966, Springer-Verlag, New York 1982.

[25] M. Gromov and R. Schoen, Harmonic maps into singular spaces and p-adic superrigidity for lattices in groups of rank one, Publ. Math. IHES 76 (1992), 165-246.

[26] G. Harder, Minkowskische Reduktionstheorie über Funktionen körpern, Inv. Math. 7 (1969), $33-54$.

[27] G. Harder, A Gauss-Bonnet formula for discrete arithmetically defined groups, Ann. Scient. Éc. Norm. Sup. 4 (1971), 409-455.

[28] J. Jost and J. Li, Finite energy and totally geodesic maps from locally symmetric spaces of finite volume, Calc. Var. 4 (1996), 409-420.

[29] J. Jost and S.-T. Yau, Harmonic maps and superrigidity, Differential Geometry: Partial Differential Equations on Manifolds, Proc. Sympos. Pure Math. 54, part 1, Amer. Math. Soc., Providence 1993, pp. 245-280.

[30] J. Jost and S.-T. Yau, Applications of quasi-linear PDE to algebraic geometry and arithmetic lattices, Algebraic Geometry and Related Topics (J. H. Yang, Y. Namikawa, and Kenji Ueno, eds.), Proceedings of International Symposium, Inchoen, Republic of Korea, International Press, Cambridge 1994, pp. 169-190.

[31] R. P. Langlands, On the functional equations satisfied by Eisenstein series, Lecture Notes in Math. 544, Springer-Verlag, New York 1976.

[32] R. P. Langlands, On the classification of irreducible representation of real algebraic groups, Representation theory and harmonic analysis on semisimple Lie groups (P. J. Sally, Jr. and D. A. Vogan, eds.), Amer. Math. Soc., Providence 1989, pp. 101-170. 
[33] E. Leuzinger, An exhaustion of locally symmetric spaces by compact submanifolds with corners, Inv. Math. 121 (1995), 389-410.

[34] R. MacPherson and M. McConnell, Explicit reduction theory for Siegel modular threefolds, Inv. Math. 111 (1993), 575-625.

[35] G. A. Margulis, Discrete groups of motions of manifolds of nonpositive curvature, A.M.S. Transl. 109 (1977), 33-45.

[36] E. Mendoza, Cohomology of $P G L_{2}$ over imaginary quadratic integers, Bonner Math. Schriften 128 (1979), Universität Bonn, Mathematisches Institut, Bonn.

[37] N. Mok, Aspects of Kähler geometry on arithmetic varieties, Several Complex Variables and Complex Geometry, Proc. Sympos. Pure Math. 52, part 2, Amer. Math. Soc., Providence 1991, pp. 335-396.

[38] N. Mok, Y.-T. Siu and S.-K. Yeung, Geometric superrigidity, Inv. Math. 113 (1993), 57-83.

[39] M. S. Osborne and G. Warner, The Selberg trace formula II: Partition, reduction, truncation, Pac. Jour. Math. 106 (1983), 307-496.

[40] M. S. Raghunathan, A note on quotients of real algebraic groups by arithmetic subgroups, Inv. Math. 4 (1968), 318-335.

[41] M. Rapoport, On the shape of the contribution of a fixed point on the boundary: The case of $\mathbb{Q}$-rank 1, The Zeta functions of Picard modular surfaces (R. P. Langlands and D. Ramakrishnan, eds.), Les Publications CRM, Montréal 1992, pp. 479-488.

[42] L. Saper, Finite energy retractions of locally symmetric spaces, preliminary version, May 6, 1993.

[43] L. Saper and M. Stern, $L_{2}$-cohomology of arithmetic varieties, Ann. of Math. 132 (1990), $1-69$.

[44] J.-P. Serre, Arbres, Amalgames, $\mathrm{SL}_{2}$, Astérisque no. 46, Soc. Math. France, Paris 1977.

[45] J.-P. Serre, Arithmetic groups, notes by Alan Robinson and Colin Maclachlan, Homological Group Theory (C. T. C. Wall, ed.), Cambridge Univ. Press, Cambridge 1979.

[46] C. Soulé, Groupes arithmétiques et $K$-théorie des anneaux d'entiers de corps de nombres, Thèse d'état, Université de Paris VII, 1978.

[47] U. Stuhler, Eine Bemerkung zur Reduktionstheorie quadratischen Formen, Archiv der Math. 27 (1976), 604-610.

[48] U. Stuhler, Zur Reduktionstheorie der quadratischen Formen II, Archiv der Math. 28 (1977), 611-619.

[49] J. Tits, Classification of algebraic semisimple groups, Algebraic groups and discontinuous subgroups (A. Borel and G. D. Mostow, eds.), Proc. Symp. Pure Math. 9, Amer. Math. Soc., Providence 1966.

[50] S. Zucker, $L_{2}$ cohomology of warped products and arithmetic groups, Inv. Math. 70 (1982), 169-218.

[51] S. Zucker, Satake Compactifications, Comment. Math. Helv. 58 (1983), 312-343.

[52] S. Zucker, $L_{2}$-cohomology and intersection homology of locally symmetric varieties, II, Comp. Math. 59 (1986), 339-398.

Leslie Saper

Department of Mathematics

Duke University

Box 90320, Durham, NC 27708 U.S.A.

and

School of Mathematics

Institute for Advanced Study

Princeton, NJ 08540 U.S.A.

E-mail: saper@math.duke.edu

(Received: May 25, 1994; revised version: September 4, 1996) 